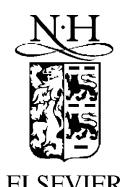

\title{
On the duality between perturbative heterotic orbifolds and M-theory on $T^{4} / Z_{N}$
}

\author{
V. Kaplunovsky a , J. Sonnenschein ${ }^{b, *}$, S. Theisen ${ }^{c}$, S. Yankielowicz ${ }^{b}$ \\ ${ }^{a}$ Theory Group, Department of Physics, University of Texas, Austin, TX 78712, USA \\ b School of Physics and Astronomy, Beverly and Raimond Sackler Faculty of Exact Sciences, \\ Tel Aviv University, Ramat-Aviv, Tel-Aviv 69978, Israel \\ c Sektion Physik, Universität München, Theresienstrasse 37, D-80333 München, Germany
}

Received 25 April 2000; accepted 19 July 2000

\begin{abstract}
The heterotic $E_{8} \times E_{8}$ string compactified on an orbifold $T^{4} / \mathbf{Z}_{N}$ has gauge group $G \times G^{\prime}$ with (massless) states in its twisted sectors which are charged under both gauge group factors. In the dual M-theory on $\left(T^{4} / \mathbf{Z}_{N}\right) \otimes\left(S^{1} / \mathbf{Z}_{2}\right)$ the two group factors are separated in the eleventh direction and the $G$ and $G^{\prime}$ gauge fields are confined to the two boundary planes, respectively. We present a scenario which allows for a resolution of this apparent paradox and assigns all massless matter multiplets locally to the different six-dimensional boundary fixed planes. The resolution consists of diagonal mixing between the gauge groups which live on the connecting seven-planes $(6 \mathrm{~d}$ and the eleventh dimension) and one of the gauge group factors. We present evidence supporting this mixing by considering gauge couplings and verify local anomaly cancellation. We also discuss open problems which arise in the presence of $U_{1}$ factors. $\odot 2000$ Elsevier Science B.V. All rights reserved.
\end{abstract}

\section{Introduction}

The rôle of M-theory for string duality is undisputed. Nevertheless, we are far from understanding this 11-dimensional theory at a fundamental level. We know that at particular points in its moduli space all known string theories are recovered. Moreover, the low energy physics is captured by 11-dimensional supergravity. Compactification and duality symmetries provide data which allow us to gain some insight into the structure of M-theory (see, e.g., Refs. [1-3] for reviews).

\footnotetext{
Research supported in part by the US-Israeli Binational Science Foundation, the US National Science Foundation (grant \#PHY-95-11632), the Robert A. Welsh Foundation, the German-Israeli Foundation for Scientific Research (GIF), by the European Commission TMR programme ERBFMRX-CT96-0045, the Israel Science Foundation and by DFG-SFB-375.

* Corresponding author.

E-mail address: cobi@post.tau.ac.il (J. Sonnenschein).
} 
In this paper we concentrate on 10-dimensional $E_{8} \times E_{8}$ heterotic string theory compactified on $T^{4} / \mathbf{Z}_{N}$ orbifolds and its dual M-theory description. The $E_{8} \times E_{8}$ heterotic string is dual to M-theory on $S^{1} / \mathbf{Z}_{2} \simeq I$. Hořava and Witten have shown [4,5] that one $E_{8}$ factor lives on each of the two boundary ten-planes ${ }^{1}$ and that the corresponding gauge multiplets are confined to it. There is no a priori M-theoretical explanation of the appearance of the $E_{8}$ gauge group on each of the two ten-planes, but they are needed for local anomaly cancellation. Thus, anomaly cancellation provides important constraints which teach us about the structure of M-theory.

Considering the proposed duality between the heterotic string on $\mathbb{R}^{5,1} \otimes\left(T^{4} / \mathbf{Z}_{N}\right)$ and M-theory on $\mathbb{R}^{5,1} \otimes\left(T^{4} / \mathbf{Z}_{N}\right) \otimes\left(S^{1} / \mathbf{Z}_{2}\right)$ one immediately encounters the following puzzle. Suppose both ten-plane $E_{8}$ groups are broken in the six-dimensional theory; let $G \times G^{\prime}$ $\subset E_{8} \times E_{8}$ denote the surviving subgroup. The twisted sectors of heterotic orbifolds generally contain massless states which are charged under both $G$ and $G^{\prime}$. In the HořavaWitten theory however, $G$ is $G$ and $G^{\prime}$ is $G^{\prime}$, they live on different ten-plains and nowhere the twain shall meet, so there does not seem to be any place where a massless state can be simultaneously charged with respect to both $G$ and $G^{\prime}$. Indeed, how would a state residing at one end of the eleventh dimension know about the gauge group acting on the other side? Somehow, in the effective seven-dimensional gauge theory on $\mathbb{R}^{5,1} \otimes\left(S^{1} / \mathbf{Z}_{2}\right)$ gauge quantum numbers ought to 'flow' from one end of the $x^{11}$ to the other end; the main objective of our work is to understand how this works.

The same problem also arises in the phenomenologically more interesting examples of orbifold compactifications to four dimensions. In fact, our initial motivation was to understand how the gauge quantum numbers work in the four-dimensional orbifolds, but the situation in six dimensions turned out to be easier. In particular, we got very useful hints from the requirement of local anomaly cancellation, which are much stronger in $d=6$ than in $d=4$. Consequently, in this article we restrict ourselves to the discussion of the six-dimensional theories with the intention to come back to the four-dimensional case in the future.

In M-theory, an $A_{n-1}$ singularity of the $\mathrm{K} 3$ compactification - such as a $\mathbf{Z}_{n}$ fixed point of an orbifold - supports an $S U_{n}$ gauge theory on the corresponding seven-plane $\mathbb{R}^{5,1} \otimes$ $\{$ f.p. $\} \otimes\left(S^{1} / \mathbf{Z}_{2}\right)$. The Cartan subalgebra arises from the zero modes of the three-form potential of 11d SUGRA and the completion to $S U_{n}$ is achieved by M2-branes wrapped on a vanishing 2 -cycle in the orbifold limit of the smooth $\mathrm{K} 3$ compactification manifold. These seven-plane gauge groups play a vital rôle in our resolution of the paradoxical $G \times G^{\prime}$ charges of the twisted states: at one end of the eleventh dimension, say at $x^{11}=0$, the seven-plane $S U_{n}$ mixes with a similar factor of $G$ - the unbroken subgroup of the $E_{8}$ living on the ten-plane boundary of the entire $11 \mathrm{~d}$ spacetime. The mixing happens along the six-planes where the fixed seven-planes intersect the $x^{11}=0$ ten-plane, but it has global consequences for the resulting effective theory: the diagonal ten-plane/seven-plane $S U_{n}$ gauge group appears to be a subgroup of the ten-plane $G$, but actually reaches along the fixed seven-planes to the other end of the $x^{11}$. Consequently, along the six-planes where the

\footnotetext{
${ }^{1}$ Throughout we refer to an extended object as a plane. E.g., a ten-plane has ten spacetime dimensions.
} 
fixed seven-planes carrying the $S U_{n}$ intersect the second ten-plane carrying $G^{\prime}$, we have both $S U_{n}$ and $G^{\prime}$ gauge fields at the same location in space - and hence the twisted states living on those six-planes may have both the $S U_{n}^{\text {diag }}$ and the $G^{\prime}$ charges in a perfectly local fashion.

The bottom line is, the twisted states have local $G^{\prime} \times S U_{n}^{7 \mathrm{P}}$ charges but from the global point of view, they have simultaneous charges under the $G^{\prime}$ and the diagonal $S U_{n}$ gauge groups. The apparent paradox of simultaneous $G^{\prime}$ and $G$ charges is due to misidentification of the diagonal ten-plane/seven-plane $S U_{n}$ as a subgroup of the ten-plane $G \subset E_{8}$. This mis-identification is natural in the perturbative heterotic string theory where the entire eleventh dimension is invisible and everything lives in ten dimensions. In the M-theory, one needs to be more careful.

In this article, we shall marshal three lines of evidence for the mixing of ten-plane and seven-plane gauge groups. First, this is the only way to reconcile the massless spectra of heterotic orbifolds with locality of the dual M-theory description. Second, the heterotic gauge couplings (which can be computed exactly in six dimensions) will show that some $S U_{n}$ gauge groups cannot be of purely perturbative origin but must be diagonally mixed with several non-perturbative factors,

$$
S U_{n}^{d=6}=\operatorname{diag}\left[S U_{n}^{\text {pert }} \times\left(S U_{n}^{\text {non-pert }}\right)^{\nu}\right],
$$

and the number $v$ of the non-perturbative $S U_{n}$ factors will always turn out to be equal to the number of fixed seven-planes in M-theory that carry $S U_{n}$ gauge groups. Finally, each sixplane in M-theory suffers from local anomalies which are sensitive to spectra of massless particles living on the six-planes themselves, on the seven-planes, on the ten-planes and in the eleven-dimensional bulk as well as inflow and intersection anomalies due to the M-theory's Chern-Simons terms. In six dimensions, it is very difficult to cancel the local anomalies unless one has correct local spectra of all the fields - and we shall see that the ten-plane/seven-plane gauge group mixing indeed provides for cancellation of all the local anomalies.

The rest of this paper is organized as follows: we begin with an illustrative example of a $\mathbf{Z}_{2}$ orbifold. In Section 2 we take a close look at the seven-plane and the ten-plane $S U_{2}$ gauge groups of this orbifold and discuss their mixing from both heterotic and M-theory points of view. In particular, we show how the mixing explains the $S U_{2}$ charges of the twisted states as well as the exact value of the $S U_{2}$ gauge coupling. In Section 3 we confirm our solution by verifying local anomaly cancellation. In these two sections we try to be as explicit as possible. Section 4 generalizes our approach to other $\mathbf{Z}_{N}$ orbifolds. The subtleties that arise in non-prime orbifolds are treated in detail.

Unfortunately, our proposed solution works for some $\mathbf{Z}_{N}$ orbifolds but has difficulties with others. Section 5 describes two common types of complications, both associated with broken seven-plane $S U_{n}$ groups. In some orbifolds (discussed in Section 5.1), the perturbative ten-plane gauge groups don't mix with the non-perturbative seven-plane groups. As far as the effective six-dimensional effective theory is concerned, the sevenplane groups are completely invisible, but the local anomalies on the six-planes are not so blind. To cancel the anomalies, we have to assume that the seven-plane gauge groups 
are not $S U_{n}$ 's but rather their Cartan subgroups $U_{1}^{(n-1)}$. Alas, from the seven-dimensional point of view, all $\mathbf{Z}_{n}$ fixed planes are created equal and we have no idea how or why does the M-theory decide that such fixed planes carry full $S U_{n}$ gauge groups in some orbifolds but only the Cartan $U_{1}^{(n-1)}$ subgroups in others. Worse problems plague orbifold models where the ten-plane and the seven-plane gauge groups do mix but the mixing involves abelian factors. In Section 5.2 we show that in such models local anomaly cancellation does not seem to work and we speculate how the seven-dimensional Chern-Simons terms might remedy this problem.

Section 6 summarizes our results. Appendices A, B and C contain some useful data about the anomalies in six dimensions and as well as some related group theory.

\section{Compactification on $\mathrm{Z}_{2}$}

\subsection{Heterotic vs. M-theory point of view}

Consider the perturbative compactification of the $E_{8} \times E_{8}$ heterotic string on the $T^{4} / \mathbf{Z}_{2}$ orbifold limit of $\mathrm{K} 3 . \mathbf{Z}_{2}$ acts as $\left(z^{1}, z^{2}\right) \rightarrow-\left(z^{1}, z^{2}\right)$ on the two complex coordinates of $T^{4}$. Under this transformation all four moduli of the torus are invariant and are thus also moduli of the orbifold. There are sixteen orbifold fixed points. This compactification has $\mathcal{N}=1$ supersymmetry in $d=6$ (eight supercharges). We represent the discrete $\mathbf{Z}_{2}$ transformation in the $E_{8} \times E_{8}$ gauge lattice via the shift vector $\delta=$ $\left(\frac{1}{2}, \frac{1}{2}, 0,0,0,0,0,0 ; 1,0,0,0,0,0,0,0\right)$. This results in the gauge group $G \times G^{\prime}=\left[E_{7}\right.$ $\left.\times S U_{2}\right] \times S O_{16} \subset E_{8} \times E_{8}$. The massless matter in the untwisted sector consists of hypermultiplets transforming as $(\underline{56}, \underline{2} ; \underline{1})$ and $(\underline{1}, \underline{1} ; \underline{128})$. They only carry charges of $G$ or $G^{\prime}$. The untwisted sector also includes the four moduli which are gauge singlets. The massless matter in the twisted sector consists of sixteen half-hyper-multiplets, one localized at each fixed point, transforming as $(\underline{1}, \underline{2} ; \underline{16})$. They carry quantum numbers under both, $G$ and $G^{\prime}$. This is the complete massless matter spectrum of this compactification. The rules to determine the massless states of heterotic K3 orbifold compactifications have recently been reviewed in [6]. The spectra of some of the models considered in this paper were constructed in [7]. We note that the difference of the number of hyper-multiplets $\left(n_{H}\right)$ and of vector-multiplets $\left(n_{V}\right)$ satisfies $n_{H}-n_{V}=244$, as required for a consistent perturbative heterotic compactification.

We want to study this compactification within the context of the conjectured duality between the heterotic string on $\mathrm{K} 3$ and M-theory on $\mathrm{K} 3 \otimes\left(S^{1} / \mathbf{Z}_{2}\right) \simeq \mathrm{K} 3 \otimes I$. In the latter description, the gauge fields of $G \times G^{\prime}$ are confined to one of the two ten-planes at the ends of the $x^{11}$ interval. We will denote them by $10 \mathrm{P}$ and $10 \mathrm{P}^{\prime}$, respectively. Since none of the perturbative gauge fields live in the bulk, it is therefore not a priori clear how the twisted matter fields, which are charged under $G$ and $G^{\prime}$, can be accommodated in the M-theory picture. From the six-dimensional point of view each of the sixteen fixed points of the heterotic compactification $T^{4} / \mathbf{Z}_{2}$ is a fixed six-plane. In the M-theory picture there are sixteen seven-planes, denoted by $7 \mathrm{P}$, of infinite extent in six spacetime directions and 
of finite extent in the $x^{11}$ direction, $x^{11} \in I=\left[0, \pi R_{11}\right]$. Their boundaries are six-planes which are the intersection of the $7 \mathrm{P}$ with the two ten-planes at $x^{11}=0$ and $x^{11}=\pi R_{11}$. It is here where the perturbative heterotic gauge groups are located, $G$ say at $x^{11}=0$ and $G^{\prime}$ at $x^{11}=\pi R_{11}$. The sixteen intersection six-planes on the $E_{7} \times S U_{2}$ side will be denoted as I6 and those on the $S_{16}$ side by I6 ${ }^{\prime}$. All the I6 are of course completely equivalent, as are the I6'.

From this geometric picture it is reasonable to expect that the 7P's do play a central role in the resolution of the puzzle with the massless twisted heterotic states in the M-theory context. They are the only objects which connect both sides of the $x^{11}$ interval. $^{2}$ On their worldvolume we have a seven-dimensional supersymmetric gauge theory.

For each $\mathbf{Z}_{2}$ fixed point of the orbifold there is an associated harmonic two-form, the Kähler form of the $S^{2}$ which has shrunk to zero size at the orbifold singularity. In M-theory on $T^{4} / \mathbf{Z}_{2}$ the three-form potential $C$ of eleven-dimensional supergravity with two internal indices and one spacetime index has thus a zero mode associated with each orbifold fixedpoint. In other words, there is a $U_{1}$ vector, and by supersymmetry, a complete sevendimensional vector-multiplet for each fixed point. ${ }^{3}$ Wrapping M2-branes around the $S^{2}$ gives rise to additional massless states in the limit of zero volume of the $S^{2}$. Taking into account two possible orientations gives $\mathrm{SU}_{2}$ as the maximal gauge group. We now compactify further to six dimensions on $S^{1} / \mathbf{Z}_{2}$. This breaks half the supersymmetry and each seven-dimensional vector-multiplet decomposes into a six-dimensional vectormultiplet $V_{7}$ and a hyper-multiplet $H_{7}$ (the subscript reminds of their seven-dimensional origin). If the five-dimensional compact manifold is the direct product $\left(T^{4} / \mathbf{Z}_{2}\right) \otimes\left(S^{1} / \mathbf{Z}_{2}\right)$, the vector components which arise from $C$ are projected out. This is because $C$ is odd under $\mathbf{Z}_{2}: x^{11} \rightarrow-x^{11}[4,5]$. In particular, the vector components must vanish on the intersection six-planes. There is no such restriction on the hyper-multiplet components.

The analysis of this model which we present in Sections 2 and 3 as well as the analysis of other models in Section 4 requires some modification of the set-up such that we retain vectors of the non-perturbative gauge group. In fact we will argue that the vectorcomponents of the full non-perturbative $S U_{2}$ survives on $\mathrm{I}^{\prime}$ and the hyper component on I6. This clearly requires a departure from the direct product geometry assumed above to a 'twisted product'. We must admit that we do not know how this works in detail. We believe that the proposed field content on any of the planes involved, in particular on the $7 \mathrm{P}$ and the I6 and I6', is correct. We give several pieces of strong evidence. Rather than being able to specify how exactly the twist acts, we can only state the effect it has on the boundary conditions of the $\mathrm{H}_{7}$ and $V_{7}$ fields. To understand what is meant here, recall that the presence of the ten-planes, or in other words, dividing by $\mathbf{Z}_{2}$, breaks half of the

\footnotetext{
${ }^{2}$ Our discussion is restricted to massless states. Their masslessness is protected by their chirality. There are massive states which are charged under both $E_{7}$ and $S O_{16}$. Since the $\mathcal{N}=1$ SUSY algebra in $d=6$ has no central charge, they are not BPS and their masses are not protected against perturbative and non-perturbative corrections. One may speculate that these states originate from open M2-branes stretched between the two tenplanes $[4,5]$. From now on we only consider massless states.

${ }^{3}$ Since $H^{2}(\mathrm{~K} 3)=22$, there are six additional vector-multiplets which are not attached to an orbifold singularity. Upon compactification, their components arrange themselves, together with components of the metric, to the supergravity multiplet, a tensor multiplet and four moduli hyper-multiplets.
} 
supersymmetries, 8 of the 16 supercharges which would be present in M-theory on K3 are even and 8 are odd under the $\mathbf{Z}_{2}$. This entails that under the $7 d \rightarrow 6 d$ decomposition the vector and the hyper components of the seven-dimensional vector-multiplet have opposite (free vs. fixed) boundary conditions at each end of the interval. The choice of the boundary conditions on both sides will be crucial below. As we shall see, we have to impose Dirichlet boundary conditions for the $S U_{2}$ vector components on the I6' and Neumann conditions on the I6. This is to be compared with the $U_{1}$ vectors which are not associated to orbifold fixed points (see the previous footnote). Here the boundary conditions are such that the zero mode for their hyper components are retained. The vector components are projected out by the $\mathbf{Z}_{2}$ twist. We want to stress once more that we are not able to derive these boundary conditions from first principles but we will present compelling arguments in favour of them.

Now that we have introduced the main ingredients of the model from the M-theory point of view, we can give a qualitative description of how they are assembled into a picture that is consistent with the heterotic description. This will involve the non-perturbative gauge groups in an essential way and we will in fact establish that the $S U_{2}$ visible in the heterotic description is the diagonal subgroup $S U_{2}^{\text {het }}=\operatorname{diag}\left[S U_{2}^{\text {pert }} \times\left(S U_{2}^{\text {non-pert }}\right)^{16}\right]$.

By looking at the heterotic spectrum of the model, it is clear that the charged states in the untwisted sector live on the ten-planes, $(\underline{56}, \underline{2} ; \underline{1})$ on $10 \mathrm{P}$ and $(\underline{1}, \underline{1} ; \underline{128})$ on $10 \mathrm{P}^{\prime}$. The major new ingredient in the M-theory description of the model is the presence of additional gauge groups, the non-perturbative $S U_{2}$ 's, one on each 7P, i.e., one for each orbifold fixedpoint. This means that we have to reconsider the $S U_{2}$ charge assignments of the fields in the twisted sector. The $E_{7}$ and the $S O_{16}$ gauge factors are unaffected by the presence of the non-perturbative $S U_{2}$ 's. The twisted matter fields necessarily live either on the I6 or I6' intersection planes. As they are charged under the $S O_{16}$, which is confined to $10 \mathrm{P}^{\prime}$, they are located on the I6' 's, one half-hyper-multiplet on each. It then also follows that we have to attribute their $S U_{2}$ quantum numbers to the non-perturbative $S U_{2}$ which lives on the $7 \mathrm{P}$ which is bounded by the I6 ${ }^{\prime}$. The situation is illustrated in the Fig. 1.

We conclude that the $i$ th twisted matter multiplet transforms as $(\underline{16}, \underline{2})$ of $\left.S O_{16}^{(\text {pert })}\right|_{10 \mathrm{P}^{\prime} \times}$ $\left.S U_{2}^{\text {(non-pert) }}\right|_{7 \mathrm{P}_{i}}$, where $i=1, \ldots, 16$. In particular it does not couple to the perturbative $S U_{2}$. For this picture to be consistent, we have to impose adequate boundary conditions for $\mathrm{H}_{7}$ and $V_{7}$. The twisted matter multiplet can couple to the gauge field only if we impose free (Neumann) boundary condition on $V_{7}$ at the $\mathrm{I}^{\prime}$ end of the 7P. As explained before, this implies fixed boundary conditions for $H_{7}$, which is thus invisible at I6'. We now have to cope with the fact that in the heterotic picture there is only one $S U_{2}$ gauge factor. This will be consistent with the M-theory description if the perturbative $S U_{2}$ which is confined to $10 \mathrm{P}$ mixes with the sixteen non-perturbative $S U_{2}$ 's such that the heterotic $S U_{2}$ is the diagonal subgroup $S U_{2}^{\text {het }}=\operatorname{diag}\left[S U_{2}^{\text {pert }} \times\left(S U_{2}^{\text {non-pert }}\right)^{16}\right]$. This requires that the $S U_{2}$ vector-multiplets $V_{7}$ are locked to the perturbative $S U_{2}$ on $10 \mathrm{P}$. That is to say that we have to impose

$$
\begin{aligned}
& A_{\mu}^{\text {non-pert }}\left(x^{1}, \ldots, x^{6}, x^{11}=0\right)=A_{\mu}^{\text {pert }}\left(x^{1}, \ldots, x^{6}, x^{7}=x^{8}=x^{9}=x^{10}=0\right), \\
& \quad \text { for } \mu=1, \ldots, 6,
\end{aligned}
$$




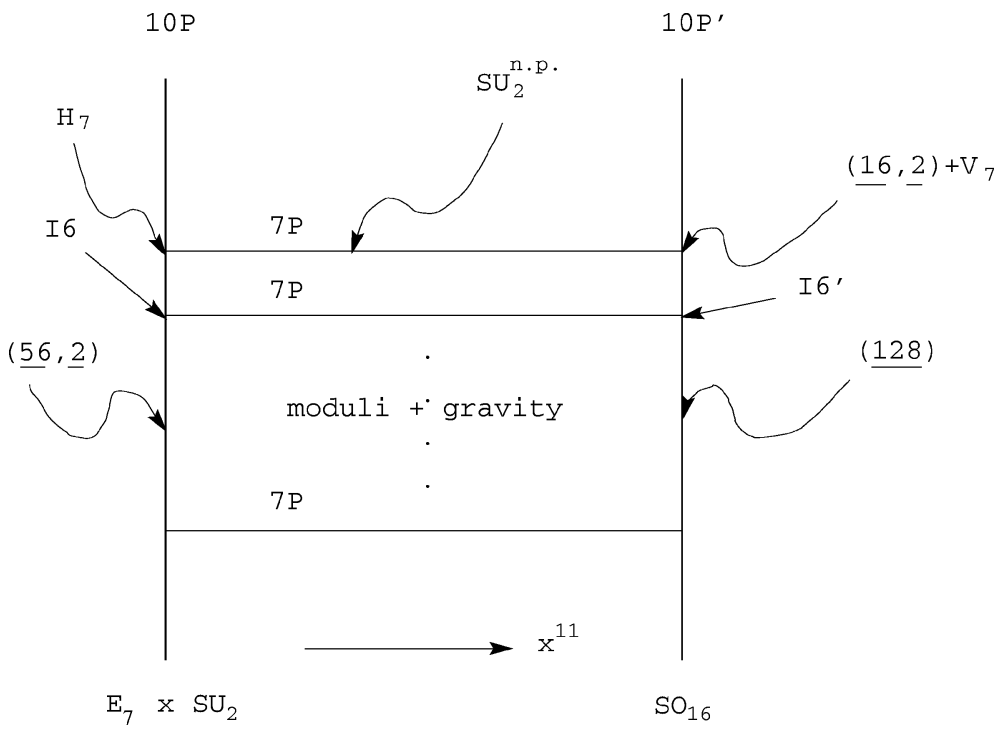

Fig. 1.

and likewise for the gauginos and at all other fixed points. Imposing fixed boundary conditions for $V_{7}$ on 10P requires free boundary conditions for the adjoint hyper-multiplet $\mathrm{H}_{7}$. Thus the latter is visible on the I6. This is also indicated in the figure.

In the following we will substantiate this picture in two independent ways. In Section 2.2 we consider the heterotic gauge couplings and we will find that indeed the $S U_{2}$ coupling has both a perturbative contribution, which is due to $\left.S U_{2}\right|_{10 \mathrm{P}}$ and a non-perturbative contribution which we interpret to arise from the sixteen $\left.S U_{2}\right|_{7 p}$ 's. In Section 2.3 we then show that the local anomalies on all intersection six-planes cancel. In particular this includes quantum contributions from either $\mathrm{V}_{7}$ or $\mathrm{H}_{7}$ and also inflow contributions from the bulk and the seven-planes. This is another check on the assignment of the fields to the different planes, as summarized in the figure.

\subsection{Consideration of the gauge couplings}

The gauge kinetic energy of the six-dimensional low-energy effective $\mathcal{N}=1 \mathrm{SYM}$ theory is, in string frame, up to a numerical constant [8]

$$
\mathcal{L} \sim \frac{1}{\alpha^{\prime}} \sum_{\alpha}\left(v_{\alpha} e^{-\phi}+\tilde{v}_{\alpha}\right) \operatorname{tr} F_{\alpha}^{2} .
$$

Here $\phi$ is the unique dilaton of the perturbative heterotic string. Additional dilatons arise if we allow for additional tensor-multiplets, but this we will not do in this paper. $e^{-\phi} \sim$ $\operatorname{Vol}(\mathrm{K} 3) / \lambda_{H}^{2} \alpha^{\prime 2}$, where $\lambda_{H}$ is the heterotic coupling constant. The sum is over all gauge group factors. $v$ and $\tilde{v}$ are dimensionless constants. For perturbative gauge groups, $v=1$ - it is in fact the level of the Kac-Moody algebra - and $\tilde{v}$ arises at one loop. For non-perturbative gauge groups, on the other hand, $v=0$ and $\tilde{v}$ is fixed at tree level. 
The coefficients $v$ and $\tilde{v}$ are related, via supersymmetry, to the coefficients of the anomaly polynomial which must factorize to allow a Green-Schwarz mechanism to cancel the anomaly. For further explanation we refer to Appendix B. Factorizability of the anomaly polynomial imposes the constraint

$$
b_{\alpha}=6\left(v_{\alpha}+\tilde{v}_{\alpha}\right),
$$

where $b_{\alpha}$ is the coefficient of the one-loop beta-function of the $d=4$ SYM theory that one obtains upon further compactification on $T^{2}$. Given the matter content of the theory we can thus compute $\tilde{v}_{\alpha}$.

The Hořava-Witten theory, at least when applied to compactifications on a smooth K3, relates $\tilde{v}$ to the net magnetic charge $k$ on the ten-plane on which it lives. The field strength $G$ of the three-from potential $C$ satisfies [8]

$$
\begin{aligned}
d G=\frac{1}{16 \pi^{2}}\{ & \delta\left(x^{11}\right)\left(\frac{1}{2} \operatorname{tr} R^{2}-\operatorname{tr} F_{1} \wedge F_{1}\right) \\
& \left.+\delta\left(x^{11}-\pi R_{11}\right)\left(\frac{1}{2} \operatorname{tr} R^{2}-\operatorname{tr} F_{2} \wedge F_{2}\right)\right\} d x^{11}
\end{aligned}
$$

from which the magnetic charge of the ten-planes is determined as $k_{1,2}=n_{1,2}-12 . n_{1,2}=$ $\frac{1}{16 \pi^{2}} \int_{\mathrm{K} 3} \operatorname{tr} F_{1,2}^{2}$ are the instanton numbers on the two sides of the interval. In perturbative compactifications, i.e., in the absence of freely floating M5-branes, $n_{1}+n_{2}=24$ and therefore $k_{1}+k_{2}=0$. Also, integrating the anomaly polynomial of the ten-dimensional heterotic theory over a smooth $\mathrm{K} 3$ one derives $\tilde{v}_{1,2}=\frac{1}{2} k_{1,2}$ and thus $\tilde{v}_{1}+\tilde{v}_{2}=0$. In particular, if two group factors arise from the same $E_{8}$, they should have the same $\tilde{v}$. For the model at hand, $n_{1}=8$ and $n_{2}=16$. Therefore, if the above analysis applied, we would find $\tilde{v}=+2$ for the $S O_{16}$ factor and $\tilde{v}=-2$ for both the $E_{7}$ and the $S U_{2}$ factor. However, using (2.3) and the field content of the theory, we find

$$
\begin{aligned}
& b\left(E_{7}\right)=-6 \rightarrow \tilde{v}\left(E_{7}\right)=-2, \\
& b\left(S U_{2}\right)=90 \quad \rightarrow \quad \tilde{v}\left(S U_{2}\right)=14 \text {, } \\
& b\left(S O_{16}\right)=18 \quad \rightarrow \quad \tilde{v}\left(S O_{16}\right)=2 .
\end{aligned}
$$

We thus realize that for the orbifold compactification the Hořava-Witten formulae work for the $E_{7}$ and the $S O_{16}$ coupling, but they do not give the correct $S U_{2}$ coupling $\tilde{v}_{S U_{2}}=$ $k / 2+16=14$. As we have argued before, the origin of the additional contribution +16 has to do with the fact that the $S U_{2}$ that we actually observe is the diagonal subgroup of the perturbative $S U_{2}$ and 16 non-perturbative $S U_{2}$ 's on the $7 \mathrm{P}$ 's.

In the heterotic theory the non-perturbative gauge fields do not contribute additional degrees of freedom but they do show up in the value for $\tilde{v}$ of the gauge factor with which they mix. The gauge coupling constant of $S U_{2}^{\text {het }}$ is thus

$$
\frac{1}{g_{\text {het }}^{2}}=\frac{1}{g_{10 \mathrm{P}}^{2}}+\sum_{i} \frac{1}{g_{7 \mathrm{P}}^{2}},
$$

where the sum is over all those non-perturbative gauge groups which mix with the perturbative gauge group on the ten-plane. All coupling constants in Eq. (2.6) are in the 
six-dimensional theory. The subscripts on the right-hand side refer to their origin. For the model considered in this section, the sum is over all sixteen non-perturbative $S U_{2}$ factors. Also, $1 / g_{10 \mathrm{P}}^{2}=1 / \alpha^{\prime}\left(\operatorname{Vol}(\mathrm{K} 3) / \lambda_{H}^{2} \alpha^{\prime 2}+\tilde{v}_{\text {pert }}\right)$ with $\tilde{v}_{\text {pert }}=k / 2$. The seven-dimensional gauge couplings are $1 /\left.g^{2}\right|_{7 \mathrm{P}} \sim R_{11} / l_{11}^{3} \sim 1 / \alpha^{\prime}$ where $l_{11}$ is the eleven-dimensional Planck length. We thus find

$$
\tilde{v}_{S U_{2}}=\frac{k_{1}}{2}+\#(7 \mathrm{P} \text { groups that mix }) .
$$

The non-perturbative contribution comes with an overall coefficient one. This coefficient was fixed using supersymmetry, which relates $\tilde{v}$ to the anomaly polynomial which gave $\tilde{v}_{S U_{2}}=-2+16$. We give further supporting evidence for this interpretation, based on the structure of the anomaly polynomial, in Appendix B.

It is now also straightforward to check that the above discussion supports the distribution of fields that we have put forward. ${ }^{4}$ The non-perturbative gauge groups mix with the perturbative $S U_{2}$ and thus must be locked to it on each I6 intersection plane. For it not to produce extra massless degrees of freedom in the heterotic limit $R_{11} \rightarrow 0$, we must impose boundary conditions such that there are no zero modes, neither for $V_{7}$ nor for $H_{7}$. Since, as discussed above, they have opposite boundary conditions (free vs. fixed) we must impose for both, the vector-multiplet and the hyper-multiplet, Neumann conditions on one end of the $x^{11}$ interval and 'Dirichlet' on the other. ${ }^{5}$ In particular $V_{7}$ then has free boundary conditions on I6. The situation for $H_{7}$ is reversed.

We have already pointed out one difference between the compactification of the heterotic string on a smooth $\mathrm{K} 3$ and on a singular K3. In the former case we always have $\tilde{v}=k / 2$ whereas this is not true in the latter case for those gauge group factors which mix with the non-perturbative gauge groups on the fixed seven-planes. Another difference between the smooth and the singular geometry is the fact that whereas in the former case the rank of the gauge group is reduced, this is not so in the latter. ${ }^{6}$ The rank reduction in the smooth case is due to the presence of a non-trivial gauge bundle with instanton numbers $n_{1,2}$. On the orbifold a $\mathbf{Z}_{n}$ singularity can support a singular $\mathbf{Z}_{n}$ instanton with instanton number $1 / n$, which breaks the gauge group without reducing its rank. E.g., for $\mathbf{Z}_{2}$, the gauge group is reduced to $\left[E_{7} \times S U_{2}\right] / \mathbf{Z}_{2}$ or $\operatorname{Spin}(16) / \mathbf{Z}_{2}$, depending on the choice of the $\mathbf{Z}_{2} \subset E_{8}$ [9]. For the model considered in this section the instanton number on the $E_{7} \times S U_{2}$ side is eight, i.e., there is one instanton of instanton number $1 / 2$ at each fixed point. On the $\mathrm{SO}_{16}$ side, we have instead one instanton of instanton number one at each fixed point.

\footnotetext{
${ }^{4}$ We should however stress that we do not have a truly M-theoretic derivation of these results.

${ }^{5}$ Note that the locking condition (2.1) is not exactly Dirichlet, but has the same effect on mode counting for the seven-dimensional fields. Both, hyper and vector components, have half-integer modes only, i.e., no zero-modes.

${ }^{6}$ This presumes a $\mathbf{Z}_{N}$ symmetric torus of generic size and no $E_{8} \times E_{8}$ Wilson lines on $T^{4}$. In the completely generic case of asymmetric orbifolds of a Narain $\Gamma_{4,20}$ compactification of the heterotic string the resulting gauge group can have rank as high as 20 or can be lower than 16 .
} 


\section{Considerations of local anomaly cancellation}

One important consistency condition that the low-energy effective field theory has to satisfy is anomaly freedom. This requirement is particularly powerful in six dimensions since in addition to pure gauge anomalies there are potential gravitational and mixed anomalies [10]. They all have to cancel. It is straightforward to verify that the anomaly of the six-dimensional field theory factorizes and the presence of one antisymmetric tensor in the massless spectrum guarantees that this anomaly can be cancelled via the GreenSchwarz mechanism.

In the M-theory description of the heterotic orbifold we have allocated all massless fields (perturbative and non-perturbative) to the bulk (gravity and moduli) and the various types of planes which are present. We can now consider the field theory on any one of these planes and since anomalies are a UV phenomenon, we need to require that they cancel locally, i.e., on any plane separately. In the bulk and on the seven-planes this is automatic, they are odd-dimensional. On each of the two ten-planes, away from the intersection sixplanes, there are 16 supercharges and an entire $E_{8}$ gauge group. Anomaly cancellation works in exactly the same way as in the Hořava-Witten theory. The situation on the intersection six-planes, however, involves new features: here supersymmetry is broken further to eight super-charges and the gauge group is broken to a subgroup. The issue of anomaly cancellation on the six-planes has to be addressed and in fact we will find that it provides a non-trivial check on the scenario advocated in Section 2.

We now turn to the evaluation of the anomaly on the intersection six-planes. It gets contributions from two sources. ( $i$ ) Quantum contributions: they arise from the massless states which are charged under the gauge group operating at the particular I6 or I6' fixed plane we are considering. Fields residing in the bulk, on the ten-plane into which the six-plane is embedded, on the seven-plane which is bounded by the six-plane and the fields confined to the six-plane do contribute. ( $i$ i Inflow and intersection contributions: they arise from gauge variance of the 11d SUGRA action. There is a contribution from a modified Bianchi identity and contributions arising from Chern-Simons (CS) terms. We first discuss the quantum contributions. Some basic results which we will be using are collected in Appendix A.

(1) Bulk fields: gravity multiplet, selfdual tensor multiplet and four moduli hypermultiplets. In this work we analyze only compactifications with one tensor multiplet in the bulk, thus restricting the discussion to the perturbative heterotic string. Recall that in the Hořava-Witten theory the gravitational anomaly is distributed evenly over the two endof-the-world ten-planes. By the same logic we distribute the contribution of the bulk fields between all $2 \times 16$ fixed I6 and I6' planes and obtain as their contribution to the anomaly on each intersection six-plane ${ }^{7}$

$$
\begin{aligned}
\mathcal{A}(\text { bulk }) & =\frac{1}{2 \cdot 16}\left[-\frac{244}{240} \operatorname{tr} R^{4}+\frac{44}{192}\left(\operatorname{tr} R^{2}\right)^{2}\right]+\frac{4}{2 \cdot 16}\left[\frac{1}{240} \operatorname{tr} R^{4}+\frac{1}{192}\left(\operatorname{tr} R^{2}\right)^{2}\right] \\
& =-\frac{1}{32} \operatorname{tr} R^{4}+\frac{1}{128}\left(\operatorname{tr} R^{2}\right)^{2} .
\end{aligned}
$$

\footnotetext{
${ }^{7}$ The correctly normalized anomaly polynomial in $d=6$ is $\left(-i / 192 \pi^{3}\right) \mathcal{A}$.
} 
The first term in the first line is the contribution from the gravity multiplet and the tensor multiplet whereas the second term is the contribution from the four moduli fields.

(2) Ten-plane fields: these are vector and hyper multiplets from the untwisted sector. We have to distinguish between I6 and I6 planes, as they are embedded in $10 \mathrm{P}$ and $10 \mathrm{P}^{\prime}$, respectively, which carry different perturbative gauge groups and untwisted matter, charged under $G$ (for I6) and $G^{\prime}$ (for $\mathrm{I}^{\prime}$ ). $\mathcal{N}=1$ supersymmetry demands that the fermions in a vector-multiplet have opposite chirality than the fermions in a hyper-multiplet and consequently they contribute to the anomaly with opposite sign. Thus generically

$$
\begin{aligned}
\mathcal{A}= & \left(n_{H}-n_{V}\right)\left(\frac{1}{240} \operatorname{tr} R^{4}+\frac{1}{192}\left(\operatorname{tr} R^{2}\right)^{2}\right) \\
& -\frac{1}{4} \operatorname{tr} R^{2}\left(\operatorname{Tr}_{H} F^{2}-\operatorname{Tr}_{V} F^{2}\right)+\left(\operatorname{Tr}_{H} F^{4}-\operatorname{Tr}_{V} F^{4}\right),
\end{aligned}
$$

where $n_{H}$ and $n_{V}$ is the number of hyper-multiplets and vector-multiplets, respectively. Distributing the anomaly of the untwisted fields charged under $E_{7} \times S U_{2}$ evenly over the 16 I6 planes, we have $H=\frac{1}{16}(\underline{56}, \underline{2})$ and $V=\frac{1}{16}[(\underline{133}, \underline{1})+(\underline{1}, \underline{3})]$. Taking into account the gauge quantum numbers we arrive, after a little algebra at ${ }^{8}$

$$
\begin{aligned}
\mathcal{A}(10 \mathrm{P})= & -\frac{1}{160} \operatorname{tr} R^{4}-\frac{1}{128}\left(\operatorname{tr} R^{2}\right)^{2}+\operatorname{tr} R^{2}\left(\frac{3}{32} \operatorname{tr} F_{E_{7}}^{2}-\frac{13}{32} \operatorname{tr} F_{S U_{2}}^{2}\right) \\
& -\frac{3}{16}\left(\operatorname{tr} F_{E_{7}}^{2}\right)^{2}+\frac{5}{16}\left(\operatorname{tr} F_{S U_{2}}^{2}\right)^{2}+\frac{9}{8} \operatorname{tr} F_{S U_{2}}^{2} \operatorname{tr} F_{E_{7}}^{2} .
\end{aligned}
$$

Similarly, at each I6 plane with $G^{\prime}=S O_{16}$ we have $H=\frac{1}{16} \underline{(128)}$ ) and $V=\frac{1}{16}(\underline{120})$, leading to

$$
\begin{aligned}
\mathcal{A}\left(10 \mathrm{P}^{\prime}\right)= & \frac{1}{480} \operatorname{tr} R^{4}+\frac{1}{384}\left(\operatorname{tr} R^{2}\right)^{2}-\frac{1}{32} \operatorname{tr} R^{2} \cdot \operatorname{tr} F_{S O_{16}}^{2} \\
& +\frac{3}{16}\left(\operatorname{tr} F_{S O_{16}}^{2}\right)^{2}-\operatorname{tr} F_{S O_{16}}^{4} .
\end{aligned}
$$

(3) Seven-plane fields: each of the sixteen 7P's connects (across the $x^{11}$ direction) an I6 plane to an $\mathrm{I}^{\prime}$ plane. As explained in the previous section, each $7 \mathrm{P}$ carries an $S U_{2}^{\text {non-pert }}$ with a $d=7$ vector-multiplet whose reduction to $d=6$ gives a vector-multiplet and an adjoint hyper-multiplet. With the assignment of boundary conditions as specified before, the hyper-multiplet component contributes to the anomaly on I6 whereas the vector-multiplet component contributes on I6'. There is however one subtlety concerning the precise contribution. A priori the contribution from both the vector and the hyper components is distributed evenly over the two bounding six-planes. The local boundary conditions however determine whether they do in fact give rise to an anomaly. This is only the case if the fields satisfy free boundary conditions. This is a manifestation of the local consistency assumption: what happens at a given boundary is sensitive only to the boundary conditions imposed there and is blind to what happens at another, distant boundary. In other words, the fact that each multiplet, due to the chosen boundary conditions, contributes to

\footnotetext{
${ }^{8}$ See Appendix $\mathrm{C}$ for the group theory involved in this derivation.
} 
the anomaly only on one of the two ends does not affect the amount by which it contributes. In summary, the contribution to the local anomaly from the 7P fields is half of that of a $S U_{2}$ vector-multiplet on I6' and half of that of an an $S U_{2}$ adjoint hyper-multiplet on I6. We then find

$$
\mathcal{A}(7 \mathrm{P})=\frac{1}{160} \operatorname{tr} R^{4}+\frac{1}{128}\left(\operatorname{tr} R^{2}\right)^{2}-\frac{1}{4} \operatorname{tr} R^{2} \cdot \operatorname{tr} F_{S U_{2}}^{2}+\left(\operatorname{tr} F_{S U_{2}}^{2}\right)^{2}
$$

on the I6 and

$$
\mathcal{A}(7 \mathrm{P})=-\frac{1}{160} \operatorname{tr} R^{4}-\frac{1}{128}\left(\operatorname{tr} R^{2}\right)^{2}+\frac{1}{4} \operatorname{tr} R^{2} \cdot \operatorname{tr} F_{S U_{2}}^{2}-\left(\operatorname{tr} F_{S U_{2}}\right)^{2}
$$

on the $\mathrm{I}^{\prime}$.

(4) Six-plane fields: these are the massless fields which are entirely confined to the I6 $\left(\mathrm{I}^{\prime}\right)$ planes. In the models which we investigate there are no such tensor or vector states and the only contribution arises from hyper-multiplets which appear in the twisted sectors of the heterotic theory. For the $\mathbf{Z}_{2}$ model considered here the twisted sector contains sixteen half-hyper-multiplets, one localized at each fixed point and transforming as $(\underline{1}, \underline{2} ; \underline{16})$. It should be assigned to the I $6^{\prime}$ side since it is charged under the gauge groups residing here, namely $\left(S U_{2} \times S O_{16}\right)$. There are no six-plane fields on I6. Note that the $S U_{2}$ quantum numbers should be understood as those pertaining to the diagonal $S U_{2}$ group. We thus find ${ }^{9}$

$$
\begin{aligned}
\mathcal{A}(6 P)= & 0, \\
\mathcal{A}\left(6 P^{\prime}\right)= & \frac{1}{15} \operatorname{tr} R^{4}+\frac{1}{12}\left(\operatorname{tr} R^{2}\right)^{2}-\operatorname{tr} R^{2}\left(\operatorname{tr} F_{S U_{2}}^{2}-\frac{1}{4} \operatorname{tr} F_{S O_{16}}^{2}\right) \\
& +\operatorname{tr} F_{S O_{16}}^{4}+\left(\operatorname{tr} F_{S U_{2}}\right)^{2}+\frac{3}{2} \operatorname{tr} F_{S U_{2}}^{2} \cdot \operatorname{tr} F_{S O_{16}}^{2} .
\end{aligned}
$$

This completes the enumeration of the quantum anomalies.

In addition to the quantum anomalies there are additional contributions which have their origin in the gauge-variance of the classical low-energy M-theory effective action [11]. More precisely, these 'inflow' contributions arise from the CS couplings $C \wedge G \wedge G$ and $C \wedge\left(\operatorname{tr} R^{4}-\frac{1}{4}\left(\operatorname{tr} R^{2}\right)^{2}\right)$ in the eleven-dimensional bulk action. Here $C$ is the three-form potential and $G$ its field strength with (magnetic) sources on the ten-planes and the sixplanes. This produces an anomaly in the gauge theory on the six-planes [11]

$$
\mathcal{A}(\text { inflow })=-g\left(\frac{1}{8} \operatorname{tr} R^{4}-\frac{1}{32}\left(\operatorname{tr} R^{2}\right)^{2}\right)-\frac{3 g}{4}\left(\operatorname{tr} F_{10}^{2}-\frac{1}{2} \operatorname{tr} R^{2}\right)^{2} .
$$

$F_{10}$ are the gauge fields on the ten planes only, i.e., $G$ or $G^{\prime}$ and $g$ is the magnetic charge of the six-plane. To the best of our knowledge the coefficients of these terms have not been reliably determined. We have fixed the normalizations such as to make the anomaly cancellation work for this model. Once fixed we use them for all other models which we

\footnotetext{
${ }^{9}$ If there were vector-multiplets confined to the six-planes, they would contribute in an obvious way. If there were $n_{T_{6}}$ tensor multiplets, there would be an additional contribution of $n_{T_{6}}\left(\frac{29}{240} \operatorname{tr} R^{4}-\frac{7}{192}\left(\operatorname{tr} R^{2}\right)^{2}\right)$.
} 
consider. ${ }^{10}$ Below we will give independent arguments in support of the normalizations in (3.7). The magnetic charges are easy to determine. We have found in Section 2.2 that the magnetic charge of the ten-planes is $k_{1,2}$. In the orbifold limit the geometric and the gauge curvatures are restricted to the orbifold singularities. Hence this is also where the magnetic charge is sitting. We thus get the following sum rule for the magnetic charges of the six-planes

$$
\sum_{i} g_{i \alpha}=k_{\alpha}
$$

The sum extends over all six-planes on a given side of the $x^{11}$ interval and $\alpha=1,2$. For the problem at hand $k_{1,2}= \pm 4$ and since all $\mathrm{I} 6$ are related by symmetry (and likewise the I $6^{\prime}$ ) we find $g_{\mathrm{I} 6}=1 / 16 \cdot(-4)=-1 / 4$ and $g_{\mathrm{I} 6^{\prime}}=1 / 16 \cdot 4=+1 / 4$. We thus obtain for the inflow contributions to the anomalies on the six-planes:

$$
\mathcal{A}(\text { inflow })=\frac{1}{32} \operatorname{tr} R^{4}-\frac{1}{128}\left(\operatorname{tr} R^{2}\right)^{2}+\frac{3}{16}\left(\operatorname{tr} F_{E_{7}}^{2}+\operatorname{tr} F_{S U_{2}}^{2}-\frac{1}{2} \operatorname{tr} R^{2}\right)^{2}
$$

on each I6 plane and

$$
\mathcal{A}(\text { inflow })=-\frac{1}{32} \operatorname{tr} R^{4}+\frac{1}{128}\left(\operatorname{tr} R^{2}\right)^{2}-\frac{3}{16}\left(\operatorname{tr} F_{S O_{16}^{2}}-\frac{1}{2} \operatorname{tr} R^{2}\right)^{2}
$$

on each I6' plane. Adding the quantum and the inflow anomalies, we obtain

$$
\mathcal{A}(\text { quantum }+ \text { inflow })=\frac{3}{2}\left(\operatorname{tr} F_{S U_{2}}^{2}-\frac{1}{16} \operatorname{tr} R^{2}\right) \cdot\left(\operatorname{tr} F_{E_{7}}^{2}+\operatorname{tr} F_{S U_{2}}^{2}-\frac{1}{2} \operatorname{tr} R^{2}\right)
$$

and

$$
\mathcal{A}(\text { quantum }+ \text { inflow })=\frac{3}{2}\left(\operatorname{tr} F_{S U_{2}}^{2}-\frac{1}{16} \operatorname{tr} R^{2}\right) \cdot\left(\operatorname{tr} F_{S O_{16}}^{2}-\frac{1}{2} \operatorname{tr} R^{2}\right) .
$$

Note that these expressions factorize into two terms, the first of a seven-plane origin and the second of a ten-plane origin.

Consistency of the theory requires, in the absence of tensor multiplets on the I6 and I6' planes, that the anomaly vanishes identically. ${ }^{11}$ Thus, were it only for these contributions to the local anomaly, the theory would be inconsistent. The factorization pattern of (3.10) does suggest yet another contribution to the anomaly. Indeed, we still have to take into account the intersection anomaly [11]. It arises from the electric coupling of the 7P to $C$. This gives rise to a CS term on each 7P worldvolume of the form $C \wedge Y_{4}$ which leads to a contribution to the anomaly on the intersection six-planes

$$
\mathcal{A}(\text { intersection })=\left(\operatorname{tr} F_{10}^{2}-\frac{1}{2} \operatorname{tr} R^{2}\right) \times Y_{4}
$$

with

\footnotetext{
${ }^{10}$ We would like to point out that our relative normalization differs by a crucial factor three from that of Ref. [11].

${ }^{11}$ In the presence of extra tensor multiplets one could invoke the GS mechanism provided the anomaly factorizes appropriately.
} 


$$
Y_{4}=\frac{3}{2}\left(\eta \operatorname{tr} R^{2}-\rho \operatorname{tr} F_{7}^{2}\right)
$$

Here $F_{7}$ refers to the $7 \mathrm{P}$ gauge fields and $F_{10}$ to the ten-plane gauge fields. $\eta$ and $\rho$ are as yet free parameters. They should follow from seven-dimensional physics and should in particular not depend on the details of the boundary conditions imposed at the ends of the interval. We will come back to this issue in Section 4. However, comparing Eqs. (3.10) and (3.11) requires that for the $\mathbf{Z}_{2}$ orbifold model considered here,

$$
\eta=\frac{1}{16} \quad \text { and } \quad \rho=1 \text {. }
$$

So far we have presented a consistent scenario for the M-theory description of the heterotic $T^{4} / \mathbf{Z}_{2}$ orbifold with gauge group $\left[E_{7} \times S U_{2}\right] \times S O_{16}$. In the remaining sections we will generalize the analysis to other $T^{4} / \mathbf{Z}_{N}$ orbifolds.

\section{Examples of $Z_{3}, Z_{4}$ and $Z_{6}$ orbifolds}

In this section we generalize the discussion of the two preceding sections to orbifolds with higher order fixed points. In Section 4.1 we recall some basic facts about $T^{4} / \mathbf{Z}_{N}$ orbifolds. We then discuss one model for $N=3,4,6$ each. We will stress the new issues which arise for non-prime orbifolds, i.e., for $N=4,6$. We first analyse the gauge couplings (Section 4.2) and then turn to the analysis of local anomalies (Section 4.3) of these models. In Section 4.4 we 'derive' the values for the parameters $\rho$ and $\eta$ in (3.12). We give two independent arguments. The first is based on a comparison between the heterotic anomaly and the M-theory anomaly. The second argument does not rely on anomaly considerations but uses the duality between M-theory on K3 and heterotic theory on $T^{3}$. To help the reader through the discussion of the various models, we have collected the data concerning their spectra, gauge couplings and magnetic charges in three tables. They can be found in Section 4.2.

\subsection{Some facts about $T^{4} / \mathbf{Z}_{N}$ orbifolds}

To ensure $d=6, \mathcal{N}=1$ supersymmetry (eight unbroken supercharges) in $T^{4} / \mathbf{Z}_{N}$ compactifications of the heterotic string, $N$ has to be restricted to $N \in\{2,3,4,6\}$. Whereas for the $\mathbf{Z}_{2}$ orbifold the number of moduli is four, for the remaining cases there are only two moduli. This follows by considering which of the $(1,1)$ forms on the torus are invariant under the $\mathbf{Z}_{N}$ twist. Recall that if $T^{4}$ is parametrized by two complex coordinates $z^{1}$ and $z^{2}$, the discrete $\mathbf{Z}_{N}$ transformation acts as $z^{1} \rightarrow \alpha z^{1}, z^{2} \rightarrow \alpha^{N-1} z^{2}$, where $\alpha^{N}=1$. The action is such that $d z^{1} \wedge d z^{2}$ survives, since this is the $(2,0)$ form which we need for a K3 compactification or the orbifold limits thereof. Its presence also guarantees at least eight unbroken supercharges. There are various ways to embed the geometrical twists as shifts in the gauge lattice, leading to different spectra. In the absence of Wilson lines there are $2(5,12,59)$ different embeddings for $\mathbf{Z}_{2}\left(\mathbf{Z}_{3}, \mathbf{Z}_{4}, \mathbf{Z}_{6}\right)$ with different gauge groups $G \times G^{\prime} \subset E_{8} \times E_{8}$ and matter content $[12,13]$. The number of fixed points is given by the Lefshetz fixed-point formula as 


$$
(1-\alpha)^{2}\left(1-\alpha^{*}\right)^{2}= \begin{cases}4^{2}, & \text { for } \mathbf{Z}_{2}, \\ 3^{2}, & \text { for } \mathbf{Z}_{3}, \\ 2^{2}, & \text { for } \mathbf{Z}_{4}, \\ 1, & \text { for } \mathbf{Z}_{6} .\end{cases}
$$

For the other possible lattice automorphisms, $N \in\{5,8,10,12\}$, the fixed point formula (4.1) does not give an integer. The precise fixed-point structure for $N$ non-prime will be discussed when we consider the $\mathbf{Z}_{4}$ and $\mathbf{Z}_{6}$ examples below.

Much of the M-theory discussion from Section 2 carries over verbatim to the general case. The fixed seven-planes are again denoted by $7 \mathrm{P}$ and they carry a non-perturbative gauge group, $G_{7}$, which for a $\mathbf{Z}_{N}$ fixed point is (at most) $S U_{N}$. The intersection six-planes are again I6 (at $x^{11}=0$ ) and I6' (at $x^{11}=\pi R_{11}$ ). There is one I6, I6 pair for each orbifold fixed point. For non-prime orbifolds there are fixed points of different orders. This leads to new features which we will discuss in detail below. But first we present another prime orbifold.

\subsection{Analysis of the gauge couplings}

\section{$\mathbf{Z}_{3}$ orbifold}

The discussion here follows very closely the discussion of the $\mathbf{Z}_{2}$ orbifold in Section 2. We will thus be brief. Since this is a prime orbifold, there is only one type of fixed points, namely nine $\mathbf{Z}_{3}$ fixed points. Consequently there are nine sevenplanes. They are bounded by nine I6 planes and nine I6' planes. The I6 are related by symmetry and are thus completely equivalent and likewise for the I6'. Two of the four moduli of the $T^{4}$ are invariant under the $\mathbf{Z}_{3}$ twist. If we choose $\delta=$ $\frac{1}{3}\left(-2,1,1,0,0,0,0,0 ; \frac{5}{2}, \frac{1}{2}, \frac{1}{2}, \frac{1}{2}, \frac{1}{2}, \frac{1}{2}, \frac{1}{2}, \frac{1}{2}\right)$ as shift vector, the gauge group is $G \times G^{\prime}=$ $\left(E_{6} \times S U_{3}\right) \times S U_{9}$. In addition to the states in the corresponding vector-multiplets, there are twist-invariant massless states which comprise hyper-multiplets transforming as $(\underline{27}, \underline{3} ; \underline{1})$ and $(\underline{1}, \underline{1} ; \underline{84})$. As for the massless twisted matter there is one hyper-multiplet $(\underline{1}, \underline{3} ; \underline{9})$ located at each of the nine fixed points. This part of the spectrum and other data pertaining to this model are summarized in Table 1.

Given the massless spectrum, it is straightforward to compute the one-loop beta-function coefficients for each group factor and from this, via (2.3), the $\tilde{v}_{\alpha}$, cf. (2.2). We find

$$
\begin{aligned}
& b\left(E_{6}\right)=-3 \quad \rightarrow \quad \tilde{v}\left(E_{6}\right)=-\frac{3}{2}, \\
& b\left(S U_{3}\right)=51 \quad \rightarrow \quad \tilde{v}\left(S U_{3}\right)=-\frac{3}{2}+9, \\
& b\left(S U_{9}\right)=15 \quad \rightarrow \quad \tilde{v}\left(S U_{9}\right)=+\frac{3}{2} .
\end{aligned}
$$

We thus learn that $\tilde{v}_{E_{7}}=k_{1} / 2$ and $\tilde{v}_{S U_{9}}=k_{2} / 2$ with $k_{1}=-3$ and $k_{2}=+3$, but $\tilde{v}_{S U_{3}}=k_{1} / 2+9$. In analogy with the $\mathbf{Z}_{2}$ example we conclude that the heterotic $S U_{3}$ gauge factor is a linear combination of the perturbative $S_{3}$ and the nine non-perturbative $S_{3}$ 's on the seven-planes; i.e., $\left(S U_{3}\right)_{\text {het }}=\operatorname{diag}\left[S U_{3}^{\text {pert }} \times\left(S U_{3}^{\text {non-pert }}\right)^{9}\right]$. Since the twisted hypermultiplets are charged under $S U_{9}$, they must be located on the $\mathrm{I}^{\prime}$ planes and they carry 
Table 1

The $\mathbf{Z}_{3}$ orbifold with gauge group $\left(E_{6} \times S U_{3}\right) \times S U_{9}$. The magnetic charges will be computed in Section 4.3, where also the notation $Q_{10}$ etc. will be explained

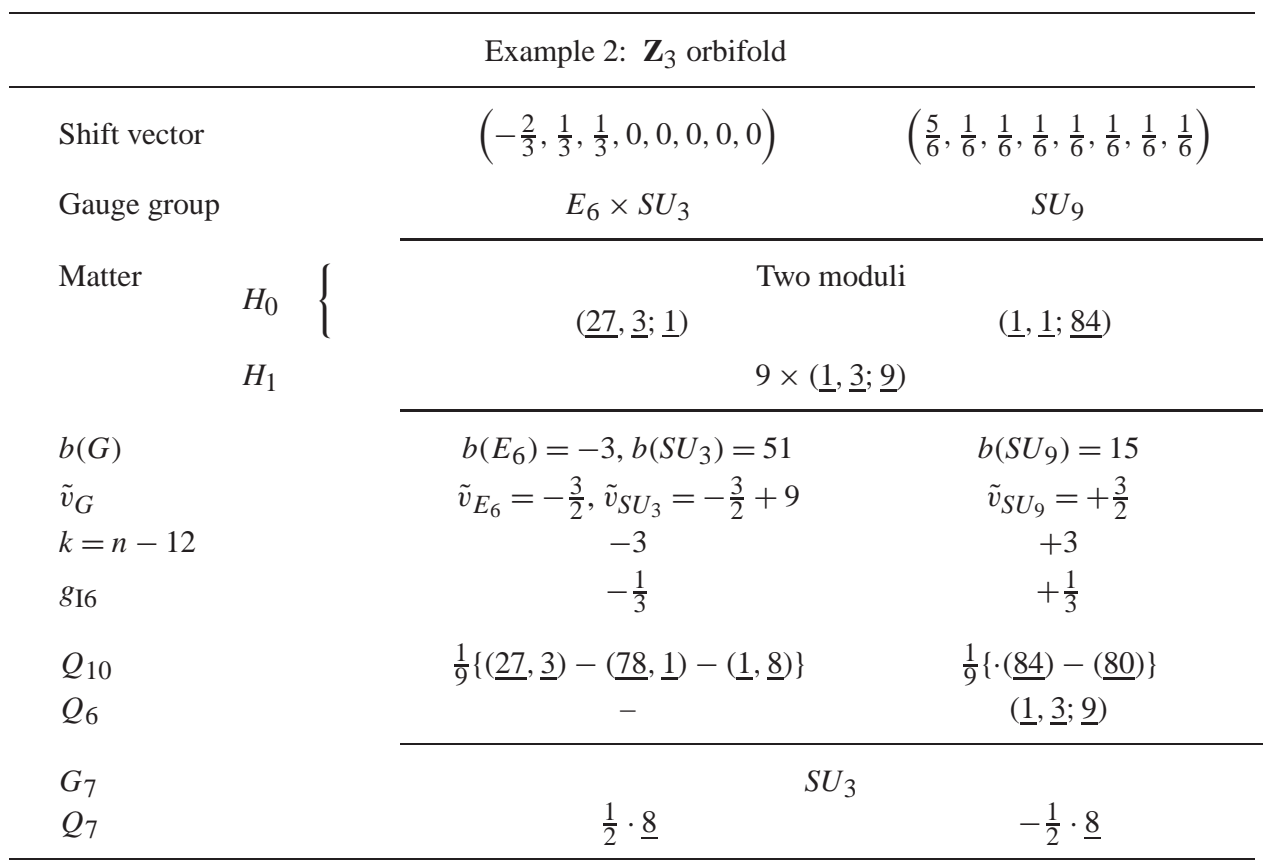

quantum numbers of a non-perturbative $S U_{3}$. The boundary conditions of the hyper and the vector components under the $7 \mathrm{~d} \rightarrow 6 \mathrm{~d}$ decomposition must be chosen such that the vector component enjoys Dirichlet boundary conditions of the type (2.1) on the $E_{6} \times S U_{3}$ side and Neumann conditions on the $S U_{9}$ side; i.e., there is one $S U_{3}$ adjoint hyper-multiplet on each I6 and one vector-multiplet on each I6'. This allocation of fields leads to local anomaly cancellation on the six-planes as we will demonstrate in Section 4.3.

\section{$\mathbf{Z}_{4}$ orbifold}

This is our first example of a non-prime orbifold. It possesses two types of fixed points: four $\mathbf{Z}_{4}$ fixed points and $16 \mathbf{Z}_{2} \subset \mathbf{Z}_{4}$ fixed-points. The latter are obviously also fixed under $\mathbf{Z}_{2}$ and the twelve remaining $\mathbf{Z}_{2}$ fixed points lie pair-wise on $\mathbf{Z}_{4}$ orbits. There are thus four $\mathbf{Z}_{4}$ and six $\mathbf{Z}_{2}$ fixed points. All fixed points of a given type are completely equivalent. The two type we have to treat separately, though. The results of the ensuing below of a particular $\mathbf{Z}_{4}$ orbifold model are collected in Table 2 .

We embed the $\mathbf{Z}_{4}$ twist into the gauge sector via the shift vector $\delta=\frac{1}{4}(-3,1,1,1,0,0,0$, $0 ;-\frac{7}{2}, \frac{1}{2}, \frac{1}{2}, \frac{1}{2}, \frac{1}{2}, \frac{1}{2}, \frac{1}{2}, \frac{1}{2}$ ), which leads to a breaking of the gauge group $E_{8} \times E_{8}$ $\rightarrow\left(S_{10} \times S O_{6}\right) \times\left(S U_{8} \times S U_{2}\right)=G \times G^{\prime}$. The massless charged matter in the untwisted sector consists of one hyper-multiplet transforming as $(\underline{16}, \underline{4} ; \underline{1}, \underline{1})$ and one hypermultiplet transforming as $(\underline{1}, \underline{1} ; \underline{28}, \underline{2})$. These states, which we denote by $H_{0}$, clearly live on $10 \mathrm{P}^{\prime}$. 
Table 2

The $\mathbf{Z}_{4}$ orbifold with gauge group $\left(S_{10} \times S U_{4}\right) \times\left(S U_{8} \times S U_{2}\right)$

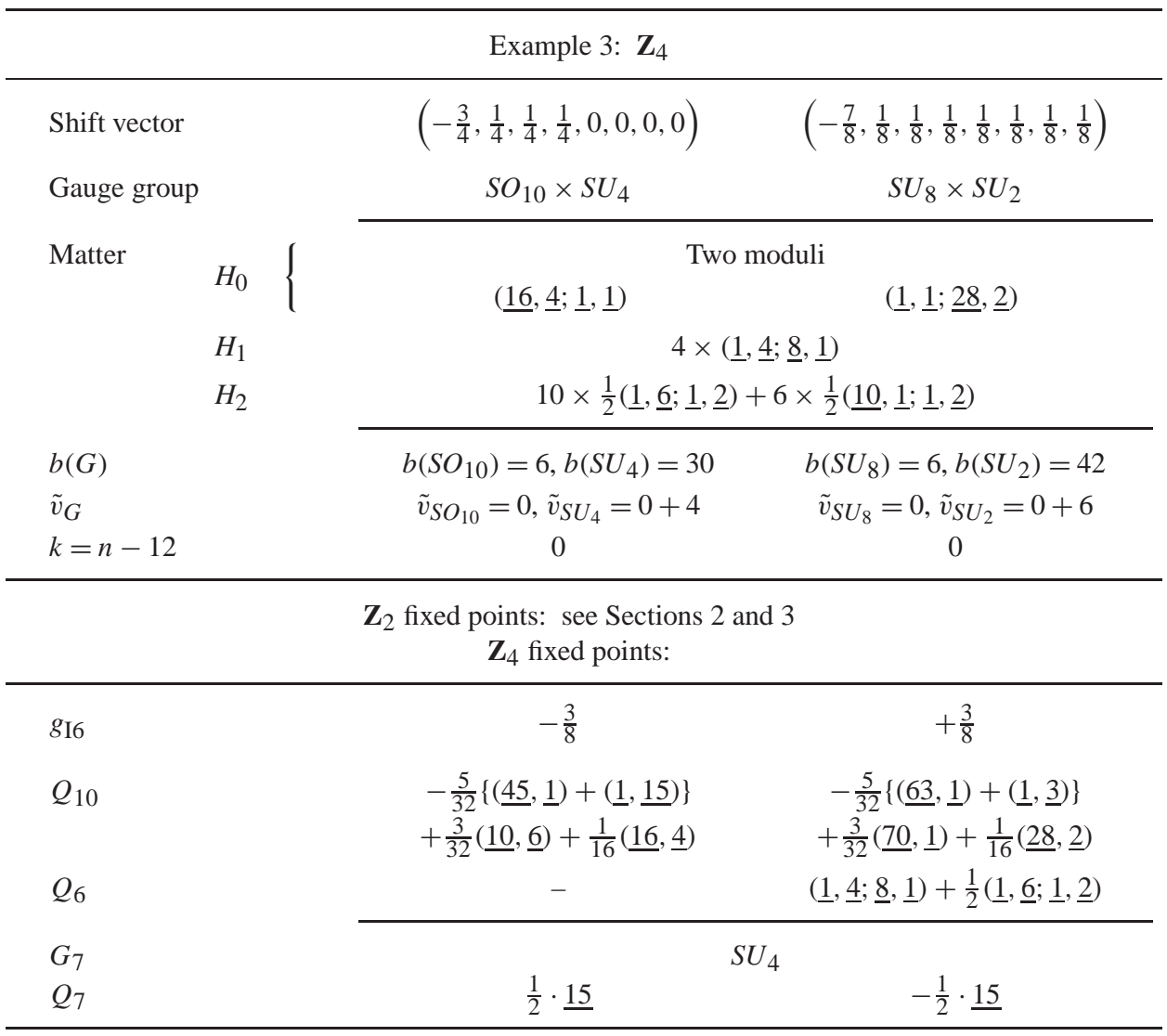

Since for non-prime orbifolds there are different types of fixed-points, one has to be careful with the allocation of the twisted matter. The $\mathbf{Z}_{4}$ orbifold has three types of twisted sectors. States in the first and the third twisted sectors combine into particle and antiparticle pairs and into complete hyper-multiplets, which we denote as $H_{1}$. These states are necessarily located at the $\mathbf{Z}_{4}$ fixed points. Straightforward application of the rules reviewed in [6] gives $H_{1}=4 \times(\underline{1}, \underline{4} ; \underline{8}, \underline{1})$. The double-twisted sector contains both particles and their anti-particles. These states we call $H_{2}$. One finds $H_{2}=10 \times \frac{1}{2}(\underline{1}, \underline{6} ; \underline{1}, \underline{2})+6$ $\times \frac{1}{2}(\underline{10}, \underline{1} ; \underline{1}, \underline{2})$. The correct assignment of these states to the various fixed points is as follows. At each $\mathbf{Z}_{2}$ fixed point: $\frac{1}{2}(\underline{1}, \underline{6} ; \underline{1}, \underline{2})+\frac{1}{2}(\underline{10}, \underline{1} ; \underline{1}, \underline{2})$ and at each $\mathbf{Z}_{4}$ fixed point: $\frac{1}{2}(\underline{1}, \underline{6} ; \underline{1}, \underline{2})$.

Note that the states at each of the $\mathbf{Z}_{2}$ fixed points combine into representations of $S O_{16} \times\left(E_{7} \times S U_{2}\right)$ which is the group left unbroken by the shift $2 \delta: \frac{1}{2}[(\underline{1}, \underline{6} ; \underline{1}, \underline{2})$ $+(\underline{10}, \underline{1} ; \underline{1}, \underline{2})]_{\left(S O_{10} \times S O_{6}\right) \times\left(S U_{8} \times S U_{2}\right)}=\frac{1}{2}(\underline{16} ; \underline{1}, \underline{2})_{S O_{16} \times\left(E_{7} \times S U_{2}\right)}$. Also, locally at the $\mathbf{Z}_{2}$ fixed points, there are four moduli hyper-multiplets. In fact, the local physics at each of the six $\mathbf{Z}_{2}$ fixed points of this $\mathbf{Z}_{4}$ orbifold is identical to that encountered in the $\mathbf{Z}_{2}$ model 
discussed before. Since there are six $\mathbf{Z}_{2}$ seven-planes, we expect $\tilde{v}_{S U_{2}}=k_{2} / 2+6$. We will verify this shortly.

It remains to discuss the four $\mathbf{Z}_{4}$ fixed points with twisted massless matter $(\underline{1}, \underline{4} ; \underline{8}, \underline{1})$ $+\frac{1}{2}(\underline{1}, \underline{6} ; \underline{1}, \underline{2})$. Each of the $\mathbf{Z}_{4}$ seven-planes supports a non-perturbative $S U_{4} \sim S O_{6}$ gauge group. This, together with the gauge quantum numbers of the twisted states, suggests that the twisted matter lives on the I6 $6^{\prime}$ planes and transforms as $\underline{4}$ and $\underline{6}$ under a non-perturbative $S U_{4}$, respectively. Also, in complete analogy to the previous examples, this would imply $\tilde{v}_{S U_{4}}=\left(k_{1} / 2\right)+4$ and thus $S U_{4}^{\text {het }}=\operatorname{diag}\left[S U_{4}^{\text {pert }} \times\left(S U_{4}^{\text {non-pert }}\right)^{4}\right]$. That this is correct can be easily verified, given that

$$
\begin{aligned}
& b\left(S O_{10}\right)=6 \quad \rightarrow \quad \tilde{v}\left(S O_{10}\right)=0, \\
& b\left(\mathrm{SO}_{6}\right)=30 \rightarrow \tilde{v}\left(\mathrm{SO}_{6}\right)=0+4 \text {, } \\
& b\left(S U_{8}\right)=6 \quad \rightarrow \quad \tilde{v}\left(S U_{8}\right)=0, \\
& b\left(S U_{2}\right)=42 \rightarrow \tilde{v}\left(S U_{2}\right)=0+6 \text {. }
\end{aligned}
$$

We also find $k_{1}=k_{2}=0$, i.e., the 24 instantons are distributed evenly over the two sides. It is now also clear how to distribute the seven-plane fields. A $S U_{4}$ adjoint hyper-multiplet lives on each of the $\mathbf{Z}_{4}$ I6 planes and a $S U_{4}$ vector-multiplet on each $\mathbf{Z}_{4}$ I6' plane.

\section{$\mathbf{Z}_{6}$ orbifold}

As in the previous examples, the data of the particular model we will discuss in this section are collected in a Table 3 which can be found at the end of this subsection.

As $\mathbf{Z}_{6}$ has two non-trivial subgroups, $\mathbf{Z}_{2}$ and $\mathbf{Z}_{3}$, a $\mathbf{Z}_{6}$ orbifold has fixed points of orders 2, 3 and 6 . The Lefshetz fixed point theorem gives one $\mathbf{Z}_{6}$ fixed point which is of course also fixed under the $\mathbf{Z}_{2}$ and $\mathbf{Z}_{3}$ subgroups. The remaining eight $\mathbf{Z}_{3}$ fixed points lie on four $\mathbf{Z}_{6}$ orbits. The $15 \mathbf{Z}_{2}$ fixed points not fixed under $\mathbf{Z}_{6}$ lie on $5 \mathbf{Z}_{6}$ orbits. A $\mathbf{Z}_{6}$ orbifold thus has one $\mathbf{Z}_{6}$, four $\mathbf{Z}_{3}$ and five $\mathbf{Z}_{2}$ fixed points. Of the four moduli of $T^{4}$, two are invariant under the $\mathbf{Z}_{6}$ twist, i.e., we have two moduli hyper-multiplets.

To proceed, we need to specify the shift vector. Our choice $\delta=\frac{1}{6}(-5,1,1,1,1,1,0,0$; $-5,1,1,1,1,1,1,1)$ leads to the gauge group $G \times G^{\prime}=\left(S U_{6} \times S U_{3} \times S U_{2}\right) \times S U_{9}$. Locally at the $\mathbf{Z}_{3}$ fixed points the gauge group is that corresponding to the shift vector $2 \delta$. One finds $\left(E_{6} \times S U_{3}\right) \times S U_{9}$ and thus recovers the situation of the $\mathbf{Z}_{3}$ orbifold model discussed above. At the $\mathbf{Z}_{2}$ fixed points the shift $3 \delta$ leads to the gauge group $\left(E_{7} \times S U_{2}\right) \times E_{8}$, i.e., the $\mathbf{Z}_{2} \subset \mathbf{Z}_{6}$ subgroup leaves the second $E_{8}$ unbroken. We will discuss this $\mathbf{Z}_{2}$ orbifold in some detail in Section 5. For the present purposes it suffices to state the following facts. The untwisted matter for this $\mathbf{Z}_{2}$ model is $H_{0}=(\underline{56}, \underline{2} ; \underline{1})$ and the twisted matter is $H_{1}=16 \times\left\{\frac{1}{2}(\underline{56}, \underline{1} ; \underline{1})+2(\underline{1}, \underline{2} ; \underline{1})\right\}$. Since the second $E_{8}$ is unbroken, all 24 instantons must sit in the first $E_{8}$, i.e., $k=12$. Finally, the $S U_{2}$ gauge-factor is purely perturbative, i.e., it does not mix with the seven-plane gauge group.

The untwisted massless matter of the $\mathbf{Z}_{6}$ orbifold consists of a single hyper-multiplet $H_{0}=(\underline{6}, \underline{3}, \underline{2} ; \underline{1})$ which lives on $10 \mathrm{P}$. The twisted matter states are $H_{1}=(\underline{6}, \underline{1}, \underline{1} ; \underline{\overline{9}})$ : this hyper-multiplet is necessarily located at the $\mathbf{Z}_{6}$ fixed point; $H_{2}=4 \times(\underline{1}, \underline{3}, \underline{1} ; \underline{9})$ : there is one such hyper-multiplet at each of the four $\mathbf{Z}_{4}$ fixed points; the states in the third-twisted 
Table 3

The $\mathbf{Z}_{6}$ orbifold with gauge group $\left(S U_{6} \times S U_{3} \times S U_{2}\right) \times S U_{9}$

\begin{tabular}{|c|c|c|}
\hline \multicolumn{3}{|c|}{ Example 4: $\mathbf{Z}_{6}$} \\
\hline Shift vector & $\left(-\frac{5}{6}, \frac{1}{6}, \frac{1}{6}, \frac{1}{6}, \frac{1}{6}, \frac{1}{6}, 0,0\right)$ & $\left(-\frac{5}{6}, \frac{1}{6}, \frac{1}{6}, \frac{1}{6}, \frac{1}{6}, \frac{1}{6}, \frac{1}{6}, \frac{1}{6}\right)$ \\
\hline Gauge group & $S U_{6} \times S U_{3} \times S U_{2}$ & $S U_{9}$ \\
\hline $\begin{array}{l}\text { Matter } \\
\end{array} H_{0}$ & $\begin{array}{ll} & \text { Two moduli } \\
(\underline{6}, \underline{3}, \underline{2} ; \underline{1}) & \end{array}$ & \\
\hline$H_{1}$ & $(\underline{6}, \underline{1}, \underline{1} ; \underline{\overline{9}})$ & \\
\hline$H_{2}$ & $4(\underline{1}, \underline{3}, \underline{1} ; \underline{9})$ & \\
\hline $\mathrm{H}_{3}$ & $\begin{array}{c}6 \times \frac{1}{2}(\underline{20}, \underline{1}, \underline{1} ; \underline{1})+5(\underline{6}, \underline{3}, \underline{1} ; \underline{1}) \\
+10(\underline{1}, \underline{1}, \underline{2} ; \underline{1})\end{array}$ & \\
\hline $\begin{array}{l}b(G) \\
\tilde{v}_{G} \\
k=n-12\end{array}$ & $\begin{array}{c}b\left(S U_{6}\right)=18, b\left(S U_{3}\right)=36, b\left(S U_{2}\right)=12 \\
\tilde{v}_{S U_{6}}=1+1, \tilde{v}_{S U_{3}}=1+4, \tilde{v}_{S U_{2}}=1 \\
2\end{array}$ & $\begin{array}{l}b\left(S U_{9}\right)=0 \\
\tilde{v}_{S U_{9}}=-1 \\
-2\end{array}$ \\
\hline \multicolumn{3}{|c|}{$\begin{array}{c}\mathbf{Z}_{2} \text { fixed points: see Section } 5 \\
\mathbf{Z}_{3} \text { fixed points: see Example } 1 \\
\mathbf{Z}_{6} \text { fixed point: }\end{array}$} \\
\hline$g_{\mathrm{I} 6}$ & $-\frac{5}{12}$ & $+\frac{5}{12}$ \\
\hline$Q_{10}$ & $\begin{array}{c}-\frac{35}{144}\{(\underline{35}, \underline{1}, \underline{1})+(\underline{1}, \underline{8}, \underline{1})+(\underline{1}, \underline{1}, \underline{3})\} \\
-\frac{5}{72}(\underline{6}, \underline{3}, \underline{2})+\frac{13}{72}(\underline{15}, \underline{3}, \underline{1})+\frac{19}{144}(\underline{20}, \underline{1}, \underline{2})\end{array}$ & $\frac{13}{72}(\underline{84})-\frac{35}{144}(\underline{80})$ \\
\hline$Q_{6}$ & - & $(\underline{6}, \underline{1}, \underline{1} ; \underline{\overline{9}})+\frac{1}{2}(\underline{20}, \underline{1}, \underline{1} ; \underline{1})$ \\
\hline$G_{7}$ & $S U_{6}$ & \\
\hline$Q_{7}$ & $\frac{1}{2} \cdot \underline{35}$ & $-\frac{1}{2} \cdot \underline{35}$ \\
\hline
\end{tabular}

sector, $H_{3}=6 \times \frac{1}{2}(\underline{20}, \underline{1}, \underline{1} ; \underline{1})+5 \times(\underline{6}, \underline{3}, \underline{1} ; \underline{1})+10(\underline{1}, \underline{1}, \underline{2} ; \underline{1})$, are assigned to the different fixed points as follows. At each of the five $\mathbf{Z}_{2}$ fixed points there is a $\frac{1}{2}(\underline{56}, \underline{1})+2(\underline{1}, \underline{2})$ hyper-multiplet of $E_{7} \times S U_{2}$. Under $E_{7} \times S U_{2} \rightarrow S U_{6} \times S U_{3} \times S U_{2}$ it decomposes as $\frac{1}{2}(\underline{20}, \underline{1}, \underline{1})+(\underline{6}, \underline{3}, \underline{1})+2(\underline{1}, \underline{1}, \underline{2})$. This leaves one $\frac{1}{2}(\underline{20}, \underline{1}, \underline{1} ; \underline{1})$ half-hyper-multiplet at the $\mathbf{Z}_{6}$ fixed points. To summarize, the massless twisted matter content at the $\mathbf{Z}_{6}$ fixed point is $(\underline{6}, \underline{1}, \underline{1} ; \underline{\overline{9}})+\frac{1}{2}(\underline{20}, \underline{1}, \underline{1} ; \underline{1})$.

The coefficients of the one-loop beta-functions and the resulting values of $\tilde{v}_{\alpha}$ are easily found to be

$$
\begin{aligned}
& b\left(S U_{6}\right)=18 \quad \rightarrow \quad \tilde{v}\left(S U_{6}\right)=1+1, \\
& b\left(S U_{3}\right)=36 \quad \rightarrow \quad \tilde{v}\left(S U_{3}\right)=1+4,
\end{aligned}
$$




$$
\begin{aligned}
& b\left(S U_{2}\right)=12 \rightarrow \tilde{v}\left(S U_{2}\right)=1, \\
& b\left(S U_{9}\right)=0 \rightarrow \tilde{v}\left(S U_{9}\right)=-1 .
\end{aligned}
$$

Since the maximal non-perturbative gauge group is $S U_{6}$, we conclude that the value of $\tilde{v}_{S U_{9}}$ must be that of a perturbative $S U_{9}$ factor. Consequently $\tilde{v}_{S U_{9}}=k_{2} / 2$ and $k_{2}=-2$, $k_{1}=+2$. From $\tilde{v}_{S U_{6}}=2$ it then follows that the non-perturbative $S U_{6}$ mixes with the perturbative $S U_{6}$, i.e., $S U_{6}^{\text {het }}=\operatorname{diag}\left[S U_{6}^{\text {pert }} \times S U_{6}^{\text {non-pert }}\right]$. The fact that $\tilde{v}_{S U_{3}}=1+4$ is in agreement with our expectation that four non-perturbative $S U_{3}$ factors located at the four $\mathbf{Z}_{3}$ seven-planes mix with the perturbative $S U_{3}$. As for $\tilde{v}_{S U_{2}}$ we would naively expect $\tilde{v}_{S U_{2}}=k_{1} / 2+5$. This would in fact be required if at the $\mathbf{Z}_{2}$ fixed points the local physics were that of the $\mathbf{Z}_{2}$ orbifold discussed in Section 2. However, as already mentioned above, for the $\mathbf{Z}_{2}$ orbifold with gauge group $\left(E_{7} \times S U_{2}\right) \times E_{8}$ we will find in Section 5 that the $S U_{2}$ factor is completely perturbative, i.e., there is no mixing with the non-perturbative seven-plane gauge group.

It is now straightforward to give the field content on the $\mathbf{Z}_{6}$ I6 and I6' planes. On I6 with perturbative gauge group $S U_{6} \times S U_{3} \times S U_{2}$ there are no twisted matter states. They are all located at $I^{\prime}$. Since they carry $S U_{6}$ quantum numbers, the non-perturbative $S U_{6}$ vector-multiplet must be free on $\mathrm{I}^{\prime}$ and the $S U_{6}$ adjoint hyper-multiplet is free on I6.

\subsection{Local anomaly cancellation}

We will now generalize the discussion of Section 3 to the models of the previous subsection. In particular we will confirm the relative normalization of the two contributions in (3.7).

\section{$\mathbf{Z}_{3}$ orbifold}

The discussion of the local anomalies for this model is almost identical to the one given in Section 3. One difference is that now we have only two moduli multiplets. Also, when distributing bulk fields and ten-plane fields over the various six-planes we have to take into account that we now have nine fixed points. With $k_{1}=-3$ and $k_{2}=+3$, the magnetic charges of the nine I6 and the nine I6 ${ }^{\prime}$ planes are $g_{\mathrm{I} 6}=-1 / 3$ and $g_{\mathrm{I} 6^{\prime}}=+1 / 3$, respectively.

It is straightforward to determine the quantum + inflow contribution to the local anomaly on the six-planes to

$$
\begin{aligned}
& \mathcal{A} \text { (quantum }+ \text { inflow on I6) } \\
& \quad=\frac{3}{2}\left(\operatorname{tr} F_{S U_{3}}^{2}-\frac{1}{9} \operatorname{tr} R^{2}\right) \cdot\left(\operatorname{tr} F_{E_{6}}^{2}+\operatorname{tr} F_{S U_{3}}^{2}-\frac{1}{2} \operatorname{tr} R^{2}\right), \\
& \left.\mathcal{A} \text { (quantum }+ \text { inflow on } \mathrm{I}^{\prime}\right) \\
& \quad=\frac{3}{2}\left(\operatorname{tr} F_{S U_{3}}^{2}-\frac{1}{9} \operatorname{tr} R^{2}\right) \cdot\left(\operatorname{tr} F_{S U_{9}}^{2}-\frac{1}{2} \operatorname{tr} R^{2}\right) .
\end{aligned}
$$

As in our discussion in Section 3, this anomaly can be cancelled by an intersection-anomaly of the form (3.11), provided that we choose the parameters in $Y_{4}$ as $\eta=1 / 9$ and $\rho=1$. 


\section{$\mathbf{Z}_{4}$ orbifold}

For this model the discussion is complicated by the fact that we have two different types of fixed points. As we have explained before, the local physics at the $\mathbf{Z}_{2}$ fixed points is identical to that of the $\mathbf{Z}_{2}$ orbifold model of Sections 2 and 3. This in particular means that anomaly cancellation on the $\mathbf{Z}_{2}$ six-planes works in exactly the same way as before. When computing the bulk and the ten-plane contribution to the anomaly on the $\mathbf{Z}_{4}$ six-planes, we must however first subtract the contribution already accounted for on the $\mathbf{Z}_{2}$ planes and then distribute the remaining anomaly over the four $\mathbf{Z}_{4}$ planes.

Before illustrating this for the charged ten-plane fields, we will introduce some convenient notation. We will denote the multiplet content of the charged ten-plane fields which contribute to the anomaly by $Q_{10}$. This splits into hyper-multiplets and vectormultiplets. Taking into account the opposite chirality of the fermions in these multiplets, we write $Q_{10}=H_{10}-V_{10}$. The net number of states will be denoted by $n_{Q_{10}}$. Let us consider the $\mathrm{SO}_{6} \times S_{10}$ side. At the $\mathrm{Z}_{2}$ fixed six-planes, the local physics is as in the $\mathbf{Z}_{2}$ model of Sections 2 and 3, i.e., the untwisted states consist of a $S O_{16}$ vector-multiplet and one $(\underline{128}) S_{16}$ hyper-multiplet. We now assume that the contribution to the anomaly of a $\mathbf{Z}_{2}$ six-plane is exactly that of such a six-plane in the $\mathbf{Z}_{2}$ orbifold, i.e., that of one sixteenth of a $\mathrm{SO}_{16}$ vector-multiplet and of one sixteenth of a 128 hyper-multiplet. Taking into account that there are six I6 of this type and four I6 fixed under $\mathbf{Z}_{4}$, we get for $Q_{10}$

$$
\begin{aligned}
Q_{10} & =\frac{1}{4}\left\{[(\underline{16}, \underline{4})-(\underline{45}, \underline{1})-(\underline{1}, \underline{15})]_{S O_{10} \times S O_{6}}-\frac{6}{16}[(\underline{128})-(\underline{120})]_{S O_{16}}\right\} \\
& =-\frac{5}{32}[(\underline{45}, \underline{1})+(\underline{1}, \underline{15})]+\frac{3}{32}(\underline{10}, \underline{6})+\frac{1}{16}(\underline{16}, \underline{4}) .
\end{aligned}
$$

In the second step we have decomposed the $\mathrm{SO}_{16}$ representation under $\mathrm{SO}_{16} \rightarrow \mathrm{SO}_{6} \times$ $S O_{10}$. Also, $n_{Q_{10}}=1 / 4$.

It follows from the construction of the states in the untwisted sector that all components of the decomposition of the $(\underline{248})_{E_{8}}$ under $E_{8} \rightarrow S O_{6} \times S O_{10}$ have definite $\mathbf{Z}_{4}$ eigenvalue, namely $e^{2 \pi i \delta \cdot P}$ where $P$ is a $E_{8}$ root and $\delta$ the shift vector in the $E_{8}$. One finds $\underline{248}=$ $(\underline{15}, \underline{1})_{+1}+(\underline{1}, \underline{45})_{+1}+(\underline{6}, \underline{10})_{-1}+(\underline{4}, \underline{16})_{+i}+(\underline{\overline{4}}, \underline{16})_{-i} \equiv \alpha(\underline{248})$. The subscripts are the $\mathbf{Z}_{4}$ eigenvalues. Here $\alpha$ denotes the $\mathbf{Z}_{4}$ generator whose action on a root of $E_{8}$ is specified by the shift vector. Introducing the function $T(x)=x / 8+x^{2} / 32$, whose argument can be either a complex number or an operator, we can rewrite (4.6) as

$$
Q_{10}=-T(\alpha) \underline{(248)} \text {. }
$$

The justification for introducing this notation is that one can define a function $T(x)$ for all $\mathbf{Z}_{N}$ orbifolds and this function is universal for any given $N$, independent of the choice of shift vector. Specifically, 


$$
T(x)= \begin{cases}\frac{x}{16}, & N=2, \\ \frac{x}{9}, & N=3, \\ \frac{x}{8}+\frac{x^{2}}{32}, & N=4, \\ \frac{x}{6}+\frac{x^{2}}{18}+\frac{x^{3}}{48}, & N=6 .\end{cases}
$$

We will need the values $T(1)=\left\{\frac{1}{16}, \frac{1}{9}, \frac{5}{32}, \frac{35}{144}\right\}$ and $2 \operatorname{Re}\left(T\left(e^{2 \pi i / n}\right)\right)=\left\{-\frac{1}{8},-\frac{1}{9}\right.$, $\left.-\frac{1}{16}, \frac{5}{72}\right\}$ for $n=\{2,3,4,6\}$, respectively. One checks that for every orbifold model we are considering, $\sum_{\mathrm{I} 6} T(1)=\sum_{\mathrm{I}^{\prime}} T(1)=1$ and $-2 \sum_{\mathrm{I} 6} \operatorname{Re}\left(T\left(e^{2 \pi i / n}\right)\right)=-2 \sum_{\mathrm{I} 6^{\prime}}$ $\times \operatorname{Re}\left(T\left(e^{2 \pi i / n}\right)\right)=\frac{1}{2} \cdot \#($ moduli), where in the case of non-prime orbifolds different types of fixed points have to be summed over.

For the $S U_{8} \times S U_{2}$ side of the $\mathbf{Z}_{4}$ orbifold one finds in a similar way

$$
\begin{aligned}
Q_{10^{\prime}}= & \frac{1}{4}\left\{[(\underline{28}, \underline{2})-(\underline{63}, \underline{1})-(\underline{1}, \underline{3})]_{S U_{8} \times S U_{2}}\right. \\
& \left.-\frac{6}{16}[(\underline{56}, \underline{2})-(\underline{133}, \underline{1})-(\underline{1}, \underline{3})]_{E_{7} \times S U_{2}}\right\} \\
= & -\frac{5}{32}[(\underline{63}, \underline{1})+(\underline{1}, \underline{3})]+\frac{3}{32}(\underline{70}, \underline{1})+\frac{1}{16}(\underline{28}, \underline{2}) \\
= & -T(\alpha)(\underline{248}),
\end{aligned}
$$

with $n_{Q_{10^{\prime}}}=-1 / 4$.

In addition to the ten-plane fields, on any six-plane there is also the contribution from the seven-plane fields with free boundary conditions on the six-plane, again with a relative sign between hyper and vector multiplets. In analogy to the notation introduced above, we will denote them as $Q_{7}$. The subscript denotes the seven-dimensional origin of these states. Including a factor of $1 / 2$, which was explained in Section 3 and denoting the fields by their $S U_{4}^{\text {non-pert }}$ representation, we have for the $\mathbf{Z}_{4}$ orbifold $Q_{7}=1 / 2 \cdot \underline{15}$ and $Q_{7^{\prime}}=-1 / 2 \cdot \underline{15}$.

As for the six-plane fields, there are none on the I6 planes. All $\mathbf{Z}_{4}$ twisted matter fields live on the I6 ${ }^{\prime}$ planes. I.e., in the by now familiar notation, $Q_{6}=\emptyset$ and $Q_{6^{\prime}}=(\underline{1}, \underline{4} ; \underline{8}, \underline{1})$ $+\frac{1}{2}(\underline{1}, \underline{6} ; \underline{1}, \underline{2})$.

If we define $Q=Q_{10}+Q_{7}+Q_{6}$ we can summarize the contribution of all charged matter fields to the quantum anomaly as

$$
\mathcal{A}(Q)=\operatorname{Tr}_{Q} F^{4}-\frac{1}{4} \operatorname{tr} R^{2} \operatorname{Tr}_{Q} F^{2}+n_{Q}\left(\frac{1}{240} \operatorname{tr} R^{4}+\frac{1}{192}\left(\operatorname{tr} R^{2}\right)^{2}\right) .
$$

With the help of the function $T$ we can also express the contribution to the anomaly of the bulk fields, namely

$$
\begin{aligned}
\mathcal{A}(\text { SUGRA }+ \text { tensor }) & =\frac{1}{2} T(1)\left[-\frac{244}{240} \operatorname{tr} R^{4}+\frac{44}{192}\left(\operatorname{tr} R^{2}\right)^{2}\right], \\
\mathcal{A}(\text { moduli }) & =-2 \operatorname{Re}\left(T\left(e^{2 \pi i / n}\right)\right)\left[\frac{1}{240} \operatorname{tr} R^{4}+\frac{1}{192}\left(\operatorname{tr} R^{2}\right)^{2}\right] .
\end{aligned}
$$


Note, e.g., that for $\mathbf{Z}_{4}$ planes, $T(1)=\frac{1}{4}(1-6 / 16)$ and $-2 \operatorname{Re}\left(T_{4}\left(e^{2 \pi i / n}\right)\right)=\frac{1}{2 \cdot 4}(2-$ $4 \cdot 6 / 16)$, where for the latter we have taken into account that a $\mathbf{Z}_{4}$ orbifold has two moduli whereas a $\mathbf{Z}_{2}$ orbifold has four moduli. (4.11) holds for both the I6 and the I6' $\mathbf{Z}_{4}$ planes.

The total quantum anomaly is thus

$$
\begin{aligned}
\mathcal{A}(\text { quantum })= & \operatorname{Tr}_{Q} F^{4}-\frac{1}{4} \operatorname{tr} R^{2} \operatorname{Tr}_{Q} F^{2} \\
& +\frac{1}{240}\left(n_{Q}-122 T(1)-2 \operatorname{Re} T\left(e^{2 \pi i / N}\right)\right) \operatorname{tr} R^{4} \\
& +\frac{1}{192}\left(n_{Q}+22 T(1)-2 \operatorname{Re} T\left(e^{2 \pi i / N}\right)\right)\left(\operatorname{tr} R^{2}\right)^{2} .
\end{aligned}
$$

This expression is valid for every $\mathbf{Z}_{N}$ six-plane of any $\mathbf{Z}_{N}$ orbifold, once $Q$ has been specified.

To compute the inflow contribution (3.7) we need the magnetic charge of the six-planes. It can be determined from the sum rule (3.8). We have to distribute the total charge $k$ over all fixed planes on a given side of the $x^{11}$ interval. For prime orbifolds they are all related by symmetry and carry the same magnetic charge. For non-prime orbifolds some care is required. E.g., on the $\mathrm{SO}_{10} \times \mathrm{SO}_{6}$ side of the $\mathbf{Z}_{4}$ model, with total charge zero $\left(k_{1}=k_{2}=0\right)$, there are six $\mathbf{Z}_{2}$ fixed planes with local gauge group $S O_{16}$, each with charge $1 / 4$. The four $\mathbf{Z}_{4}$ fixed planes must thus carry a total charge of $-6 \cdot 1 / 4$ or $-3 / 8$ each. I.e., $g_{\mathrm{I} 6}=-3 / 8$ and $g_{\mathrm{I} 6^{\prime}}=+3 / 8$.

Note that there is a minimal magnetic charge any fixed plane must carry. This is obtained if $k=-12$, which corresponds to an unbroken $E_{8}$. In this case there are no gauge instantons. It is straightforward to compute the minimal magnetic charges of the various $\mathbf{Z}_{n}$ planes. They can be conveniently summarized in the formula $g_{\mathbf{Z}_{n}}^{\min }=\left(1-n^{2}\right) / 2 n$. The allowed magnetic charges are then $g_{\mathbf{Z}_{n}}=g+m / n$ where the non-negative integer $m$ counts the number of $\mathbf{Z}_{n}$ instantons sitting at the fixed point.

We have now provided all ingredients necessary to compute the quantum and the inflow anomaly for the $\mathbf{Z}_{4}$ model. A short calculation gives

$$
\begin{aligned}
& \mathcal{A} \text { (quantum }+ \text { inflow on I6) } \\
& \quad=\frac{3}{2}\left(\operatorname{tr} F_{S U_{4}}^{2}-\frac{5}{32} \operatorname{tr} R^{2}\right) \cdot\left(\operatorname{tr} F_{S O_{10}}^{2}+\operatorname{tr} F_{S O_{6}}^{2}-\frac{1}{2} \operatorname{tr} R^{2}\right), \\
& \mathcal{A} \text { (quantum }+ \text { inflow on I6') } \\
& \quad=\frac{3}{2}\left(\operatorname{tr} F_{S U_{4}}^{2}-\frac{5}{32} \operatorname{tr} R^{2}\right) \cdot\left(\operatorname{tr} F_{S U_{8}}^{2}+\operatorname{tr} F_{S U_{2}}^{2}-\frac{1}{2} \operatorname{tr} R^{2}\right) .
\end{aligned}
$$

We once again find that this anomaly can be cancelled via (3.11) with $\rho=1$ and $\eta=$ $T(1)=5 / 32$.

\section{$\mathbf{Z}_{6}$ orbifold}

We will be very brief here. We only have to check anomaly cancellation on the $\mathbf{Z}_{6}$ sixplanes. The ten-plane gauge groups are $S U_{6} \times S U_{3} \times S U_{2}$ on $10 \mathrm{P}$ and $S U_{9}$ on $10 \mathrm{P}^{\prime}$. Using $T(x)$ as given in (4.8) it is straightforward to show that 


$$
\begin{aligned}
Q_{10}= & -T(\alpha)(\underline{248}) \\
= & -\frac{35}{144}[(\underline{35}, \underline{1}, \underline{1})+(\underline{1}, \underline{8}, \underline{1})+(\underline{1}, \underline{1}, \underline{3})]-\frac{5}{72}(\underline{6}, \underline{3}, \underline{2}) \\
& +\frac{13}{72}(\underline{15}, \underline{\overline{3}}, \underline{1})+\frac{19}{144}(\underline{20}, \underline{1}, \underline{2}), \\
Q_{10^{\prime}}= & \frac{13}{72} \cdot \underline{84}-\frac{135}{144} \cdot \underline{80},
\end{aligned}
$$

where the decomposition is with respect to the ten-plane gauge groups. Also, the discussion in Section 4.1 gave

$$
Q_{6}=\emptyset, \quad Q_{6^{\prime}}=(\underline{6}, \underline{1}, \underline{1} ; \underline{\overline{9}})+\frac{1}{2}(\underline{20}, \underline{1}, \underline{1} ; \underline{1})
$$

and

$$
Q_{7}=\frac{1}{2} \cdot \underline{35}, \quad Q_{7^{\prime}}=-\frac{1}{2} \cdot \underline{35},
$$

where the latter states are with respect to $S U_{6}^{\text {non-pert }}$. The magnetic charges are easily determined to $g_{\mathrm{I} 6}=-5 / 12$ and $g_{\mathrm{I} 6^{\prime}}=+5 / 12$.

For both the I6 and the I6 ${ }^{\prime}$ planes, the quantum + inflow anomaly can be cancelled via an intersection anomaly (3.11) with $\rho=1$ and $\eta=T(1)=35 / 144$.

\subsection{Common features}

We have demonstrated in all four examples that the local anomaly on each intersection six-plane cancels. This is to say that in the sum of quantum + inflow + intersection anomaly the coefficients of $\operatorname{tr} R^{4}$, $\left(\operatorname{tr} R^{2}\right)^{2}, \operatorname{tr} R^{2}$ and of the term without dependence on the Ricciform vanish separately. The four conditions for this to happen are:

$$
\begin{aligned}
n_{Q} & =122 T(1)+T\left(e^{2 \pi i / n}\right)+T\left(e^{-2 \pi i / n}\right)+30 g, \\
\eta & =T(1), \\
\frac{1}{3} \operatorname{Tr}_{Q} F^{2} & =\rho \operatorname{tr} F_{7}^{2}+(g+2 \eta) \operatorname{tr} F_{10}^{2}, \\
\frac{2}{3} \operatorname{Tr}_{Q} F^{4} & =\operatorname{tr} F_{10}^{2}\left(\frac{g}{2} \operatorname{tr} F_{10}^{2}+\rho \operatorname{tr} F_{7}^{2}\right) .
\end{aligned}
$$

In particular, local anomaly cancellation fixed the coefficients $\eta$ and $\rho$ in (3.12) to $\eta=T$ (1) and $\rho=1$, respectively. There is another way to see why these values are generic, which we will now present. It also uses anomaly arguments and it involves a direct comparison of the heterotic and the M-theory point of views.

In the heterotic theory, anomaly cancellation is of course guaranteed by the wellestablished consistency of the perturbative heterotic string. However, by realizing that the massless fields which contribute to the heterotic anomaly on a given fixed six-plane are precisely those which, in M-theory, contribute on a I6, I6' pair which is connected by a seven-plane, we can, by comparison, determine the coefficients $\eta$ and $\rho$.

The boundary conditions of the seven-plane fields were chosen such that in the limit $R_{11} \rightarrow 0$ there are no additional massless states. This is reflected in $Q_{7}+Q_{7}^{\prime}=0$. Also, 
the magnetic charges satisfy $g_{\mathrm{I} 6}+g_{\mathrm{I} 6^{\prime}}=0$. We then have $Q_{\text {net }} \equiv Q+Q^{\prime}=Q_{10}+Q_{10}^{\prime}$ $+Q_{6}+Q_{6}^{\prime}$ and the first condition in (4.17) gives $n_{Q_{\text {net }}}=244 T(1)+4 \operatorname{Re} T\left(e^{2 \pi i / n}\right)$, which is correct in the heterotic context without any reference to M-theory.

We can now compute the anomaly on a I6, I6' pair from the heterotic and from the M-theoretic point of view. The former gives (cf. Appendix B)

$$
\begin{aligned}
\frac{2}{3} \mathcal{A} & =\frac{2}{3} \operatorname{Tr}_{Q_{\text {net }}} F^{4}-\frac{1}{6} \operatorname{tr} R^{2} \operatorname{Tr}_{Q_{\text {net }}} F^{2}+T(1)\left(\operatorname{tr} R^{2}\right)^{2} \\
& =\left(\operatorname{tr} R^{2}-\sum_{\alpha} \operatorname{tr} F_{\alpha}^{2}\right) \wedge\left(T(1) \operatorname{tr} R^{2}-\sum_{\alpha} \tilde{v}_{\alpha} \operatorname{tr}_{\alpha} F^{2}\right) .
\end{aligned}
$$

Computing the same anomaly in the M-theory picture produces instead (use the last two equations in (4.17))

$$
\frac{2}{3} \mathcal{A}=\left(\operatorname{tr} R^{2}-\operatorname{tr} F_{10}^{2}-\operatorname{tr} F_{10^{\prime}}^{2}\right) \wedge\left(\eta \operatorname{tr} R^{2}+\frac{g}{2}\left(\operatorname{tr} F_{10}^{2}-\operatorname{tr} F_{10^{\prime}}^{2}\right)+\rho \operatorname{tr} F_{7}^{2}\right) .
$$

Comparison gives once more $\eta=T(1)$ and

$$
\begin{aligned}
& v_{10}=v_{10^{\prime}}=1 \quad \text { and } \quad \tilde{v}_{10}=\frac{1}{2} \sum_{\substack{\text { fixed } \\
\text { planes }}} g=-\tilde{v}_{10^{\prime}}, \\
& v_{7}=0 \quad \text { and } \quad \tilde{v}_{7}=\rho .
\end{aligned}
$$

The seven-plane gauge groups $G_{7}$ thus have the characteristic of non-perturbatively generated gauge groups. However, in the heterotic dual, which is completely perturbative, they are not visible as additional gauge group factors. This leaves two options: $(i) \rho \neq 0$ and $\rho$ is the level of the gauge group $G_{7}$. This is the situation we have encountered in all four examples considered so far. The seven-plane gauge group mixes with the ten-plane gauge group, as, e.g., in (2.7). ( $i$ i ) $\rho=0$ : this case will be discussed in Section 5.

So far we have determined the parameters $\rho$ and $\eta$ using anomaly arguments. However, ultimately these parameters should come from seven-dimensional physics. In fact, we will now give an independent 'derivation' of the values for $\eta$ and $\rho$, which does not rely on any anomaly arguments.

In M-theory on $\mathrm{K} 3$, the parameters $\eta$ and $\rho$ enter through the electric coupling of the seven-plane to the three-form potential $C$ of 11-dimensional supergravity via the term $C \wedge Y_{4}$, cf. (3.12) and [11]. The eleven-dimensional origin of this term are the two CS terms $C \wedge G \wedge G$ and $C \wedge\left(\operatorname{tr} R^{4}-\frac{1}{4}\left(\operatorname{tr} R^{2}\right)^{2}\right)$. However, it is easier to discuss these couplings from the dual point of view, exploiting the duality between M-theory on $\mathrm{K} 3$ and the heterotic theory on $T^{3}[1,14] .^{12}$

This heterotic - M-theory duality in $d=7$ relates the field strength of $C$, denoted by $G$, to the field strength $H$ of the Kalb-Ramond field $B$ of the heterotic theory and vice versa: $H \leftrightarrow * G$. The moduli spaces of the heterotic compactification on $T^{3}$ and of M-theory on $\mathrm{K} 3$ are isomorphic. At a generic point on the Narain lattice, the gauge symmetry of

\footnotetext{
12 This seven-dimensional heterotic theory is of course completely different from the Hořava-Witten theory we have discussed so far.
} 
the heterotic string compactified on $T^{3}$ is $U(1)^{22}$ which is also the gauge group of the M-theory at a generic point of the K3 moduli space. On the heterotic side, the Bianchi identity reads

$$
d H \propto \operatorname{tr} R^{2}+\sum_{I, J=1}^{22} d_{I J} F_{I} F_{J},
$$

where $d_{I J}$ is a Lorentzian metric with signature $\left((+)^{3},(-)^{19}\right)$ which is also the signature of the intersection matrix of the K3 homology 2-cycles. Duality now implies $d(* G) \propto d H$ and thus an electric coupling $\propto C \wedge d H$. At special points in the Narain moduli space the heterotic gauge symmetry acquires non-Abelian components which contribute to $d H$, i.e., (4.21) gets modified to

$$
d H \propto \operatorname{tr} R^{2}-\sum \operatorname{tr} F_{i}^{2}+\text { Abelian part. }
$$

For the M-theory the gauge group enhancement happens at the orbifold points of the K3 moduli space. The additional states which comprise the non-Abelian gauge multiplets are provided by M2-branes wrapping the vanishing cycles (cf. the discussion in Section 1). At the orbifold points the seven-dimensional gauge groups are $S U_{2}^{16} \times U_{1}^{6}$ for the $\mathbf{Z}_{2}$ orbifold, $S U_{3}^{9} \times U_{1}^{4}$ for $\mathbf{Z}_{3}, S U_{2}^{6} \times S U_{4}^{4} \times U_{1}^{4}$ for $\mathbf{Z}_{4}$ and $S U_{2}^{5} \times S U_{3}^{4} \times S U_{6} \times U_{1}^{4}$ for $\mathbf{Z}_{6}$.

From the eleven-dimensional point of view the Abelian part lives in the bulk and is completely broken after compactification to six-dimensions. It is thus of no further interest for us. The non-Abelian part, on the other hand, contributes to the local $C \wedge Y_{4}$ coupling on the seven-planes as $-C \wedge \sum_{i} \operatorname{tr} F_{7 i}^{2}$. The sum is over all fixed seven-planes. As far as the $C \wedge \operatorname{tr} R^{2}$ piece of this coupling is concerned, it has to be apportioned between all sevenplanes. The apportioning happens in exactly the same manner as with the bulk anomaly in previous sections, namely as $T(1) \operatorname{tr} R^{2}$. The reason for this is the same as before. The contribution on a $\mathbf{Z}_{N}$ plane depends only on $N$, independent of the orbifold model.

We thus find that the relevant coupling is proportional to

$$
C \wedge\left(\operatorname{tr} R^{2}-\sum_{i} \operatorname{tr} F_{7 i}^{2}\right)=C \wedge \sum_{i}\left(T_{i}(1) \operatorname{tr} R^{2}-\operatorname{tr} F_{7 i}^{2}\right)
$$

Comparing this with $C \wedge \sum_{i} Y_{4 i}$ leads to the identification $\eta=T(1)$ and $\rho=1$.

We should point out that we have not been careful with the overall normalization of the $C \wedge Y_{4}$ coupling. In fact, this coupling is somewhat controversial since it is related to the normalization of the two eleven-dimensional CS terms. Nevertheless the normalization must be universal and it can be fixed by considering any orbifold model, such as the $\mathbf{Z}_{2}$ model of Sections 2 and $3 .^{13}$

\footnotetext{
${ }^{13}$ If one changed the relative normalization of the two terms in (3.7) one would find that the second of the conditions in (4.17) will be modified ( $\eta$ and $T$ (1) will no longer be proportional to each other), in contradiction with the independent arguments given here.
} 


\section{Open problems}

All the orbifold models we have discussed thus far followed a common pattern from the M-theory point of view: each $\mathbf{Z}_{n}$ fixed seven-plane carries a non-perturbative $S U_{n}$ gauge theory which mixes with a perturbative gauge theory living on one of the two ten-planes. The resulting theory contains an $S U_{n}$ gauge group which appears to be a subgroup of the $G \subset E_{8}$ (or $G^{\prime} \subset E_{8}^{\prime}$ ) but isn't actually confined to one side of the $x^{11}$ interval. Instead, it reaches to the other side along the fixed seven-planes - and that's how the twisted states living on the I6' intersections manage to have charges under both the $S U_{n} \subset G$ and the $G^{\prime}$ gauge groups.

However, in many other orbifold models, the non-perturbative gauge groups living on the $\mathbf{Z}_{n}$ fixed seven-planes turn out to be proper subgroups $G_{7} \subset S U_{n}$ rather that complete $S U_{n}$ 's. Furthermore, such reduced non-perturbative groups - or some of their factors do not mix with the perturbative gauge group factors but simply decouple from the massless states of the six-dimensional theory. ${ }^{14}$ At present, we do not know any M-theoretical rules governing breaking of the non-perturbative gauge groups or their mixing with the perturbative gauge group factors. All we have are the 'experimental data' about the nonperturbative gauge groups implied by the quantum numbers of the twisted states in a score of orbifold models we have studied in some detail.

We see no point in boring the reader with technical details of too many models. Instead, we shall simply present examples of two common problems we have seen in several models. In the following Section 5.1 we discuss models with the unbroken $E_{8}^{\prime}$ gauge group. 'Experimentally', all such models have $G_{7}=\left(U_{1}\right)^{(n-1)}$, the Cartan subgroup of the $S U_{n}$, and none of the non-perturbative $U_{1}$ factors mixes with any perturbative gauge groups. Furthermore, the local anomaly cancellation requires the non-perturbative $U_{1}$ 's to have $\rho=0$, in blatant contradiction with the seven-dimensional arguments of Section 4.4.

In Section 5.2 we present an example of a more complicated model where the combined quantum, inflow and intersection anomalies do not cancel out for any $G_{7}$ consistent with the twisted states' quantum numbers. This problem occurs in all models with mixed perturbative/non-perturbative Abelian gauge fields, although it may affect the non-Abelian fields as well. We speculate how seven-dimensional Chern-Simons couplings may solve this problem, but a thorough analysis has to be postponed to a later publication.

\subsection{Models with an unbroken $E_{8}$}

To exemplify the problems that arise in this class of models, consider the best known heterotic orbifold, namely the $\mathbf{Z}_{2}$ orbifold with the standard imbedding of the spin connection into the gauge group, i.e., $\delta=\left(-\frac{1}{2},+\frac{1}{2}, 0,0,0,0,0,0 ; 0,0,0,0,0,0,0,0\right)$. In this model, the first $E_{8}$ is broken down to $G=E_{7} \times S U_{2}$ while the second $E_{8}$ remains unbroken. Note that we have already encountered such $\mathbf{Z}_{2}$ fixed points in the $\mathbf{Z}_{6}$ orbifold

\footnotetext{
14 Technically, the locking boundary conditions (2.1) at one side of the $x^{11}$ interval are replaced with the simple Dirichlet boundary conditions $A_{\mu}^{\text {non-pert }}\left(x^{11}=0\right)=0$.
} 
Table 4

The $\mathbf{Z}_{2}$ orbifold with the $\left(E_{7} \times S U_{2}\right) \times E_{8}$ gauge group

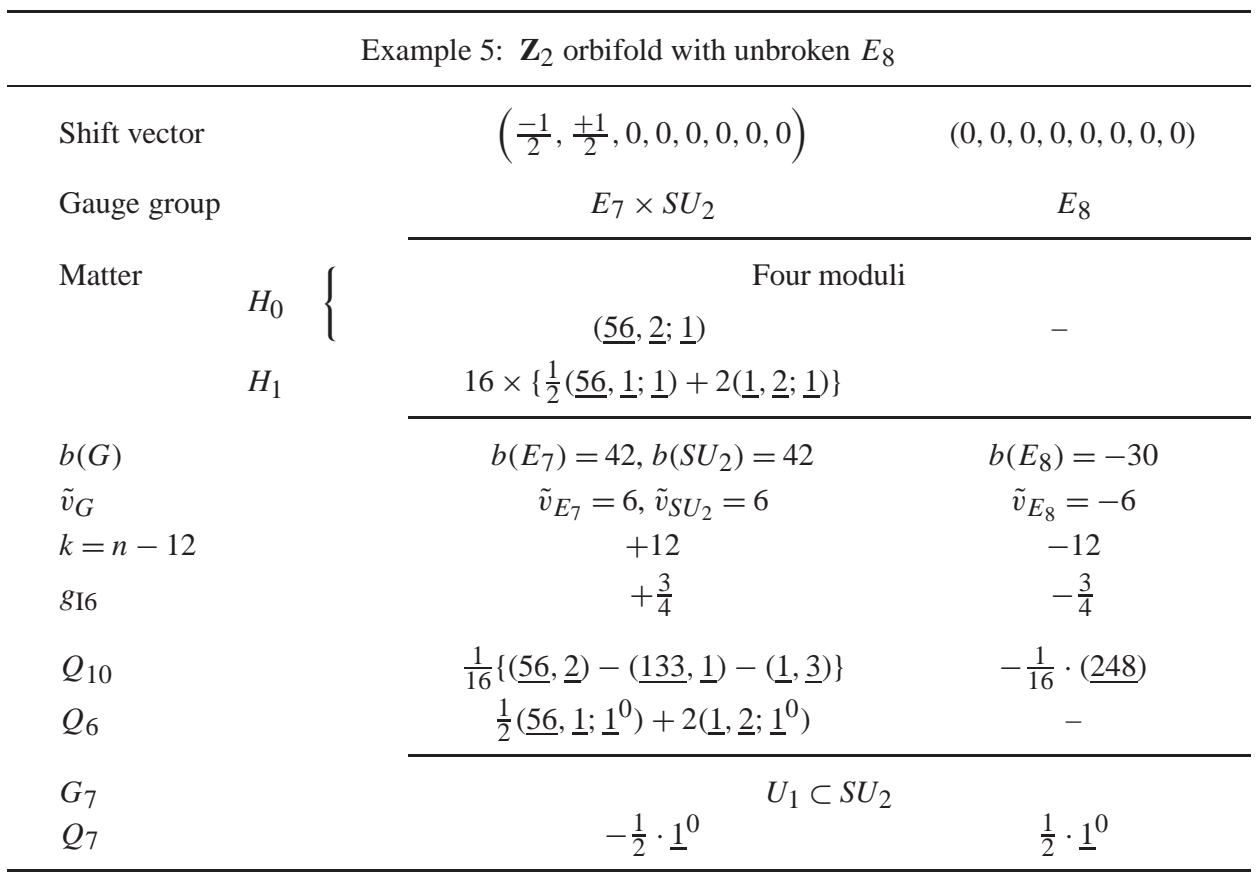

in Section 4, but let us take a closer look now. The hypermultiplet spectrum and other technical details of the 'standard' $\mathbf{Z}_{2}$ orbifold are summarized in Table 4.

Note that unlike the 'non-standard' $\mathbf{Z}_{2}$ orbifold discussed in Section 2, the 'standard' $\mathbf{Z}_{2}$ orbifold has $\tilde{v}=k / 2$ for all gauge group factors including $S U_{2}$; indeed, on the $E_{7} \times S U_{2}$ side, we have $k=+12$ and $\tilde{v}_{S U_{2}}=6$ rather than $\tilde{v}_{S U_{2}}=6+16=22$. According to Eq. (2.7), this indicates that in the standard $\mathbf{Z}_{2}$ orbifold, the $S U_{2}$ gauge factor is purely perturbative and does not mix with any non-perturbative factors. Consequently, no massless states in this model can be simultaneously charged under the $S U_{2}$ and the $E_{8}$ gauge groups — and indeed there are no such states in the standard orbifold.

The non-perturbative gauge group $G_{7}$ on each of the 16 fixed seven-planes must fit inside an $S U_{2}$ (bigger groups are not available at $A_{1}$ singularities such as $\mathbf{Z}_{2}$ fixed points), but because it does not mix with the perturbative $S U(2)$, we may have either $G_{7}=S U_{2}$ or $G_{7}=U_{1}$. The choice of $G_{7}$ affects the $Q_{7}$ contribution to the quantum anomaly at each end of the $x^{11}$ : given our general rule of opposite boundary conditions on opposite ends (two avoid non-perturbative massless states in $6 \mathrm{~d}$ ) and the requirement for free vector fields at either end to form a closed subgroup of the $G_{7}$, it follows that $n\left(Q_{7}^{(\prime)}\right)=\mp 3 / 2$ for $G_{7}=S U(2)$ but $n\left(Q_{7}^{(\prime)}\right)=\mp 1 / 2$ for $G_{7}=U_{1}$. Since the $Q_{6}^{(\prime)}$ and $Q_{10}^{(\prime)}$ contributions to the anomaly on each side are completely fixed by the massless spectrum of the orbifold, and since $k= \pm 12$ implies $g= \pm 3 / 4$, the need to cancel the $\operatorname{tr} R^{4}$ term in the local anomaly at each end (cf. first Eq. (4.17)) requires $n\left(Q_{7}\right)=-1 / 2$ on the $E_{7} \times S U_{2}$ side 
and $n\left(Q_{7}^{\prime}\right)=+1 / 2$ on the $E_{8}$ side — and hence $G_{7}=U_{1} \subset S U_{2}$ rather than $G_{7}=S U(2)$.

Without going into any more details of Eqs. (4.17), let us simply state that for $G_{7}=U(1)$, the entire local anomaly polynomials $\mathcal{A}$ on both I6 and I6' planes cancel, provided (1) all the twisted states $Q_{6}$ are neutral with respect to the non-perturbative $U_{1}$ and (2) $\rho=0$ in the intersection anomaly term, all the arguments in Section 4.4 for $\rho=1$ notwithstanding; note however that $\eta=1 / 16=T(1)$ as required by the second Eq. (4.17). At present, we have no explanation for the 'experimental fact' of $\rho=0$ except that its required to cancel the anomalies locally. Likewise, we have no M-theoretical explanation for the $G_{7}=U_{1}$, only the brute fact that this too is required to cancel the anomalies locally. The theory would have to wait for a later publication.

In lieu of theory, we offer a summary of 'experimental' data to show a common pattern of invisible non-perturbative Abelian gauge groups, which do not mix with any perturbative gauge group factors and don't couple to any twisted massless states (i.e., the hypermultiplets living on I6 and I6' are neutral with respect to the invisible groups). In particular, in all $\mathbf{Z}_{n}$ orbifolds ( $\left.n=2,3,4,6\right)$ with an unbroken $E_{8}$ group ${ }^{15}$ we can cancel all anomalies locally, provided: (1) each $G_{7}\left(\mathbf{Z}_{n}\right.$ plane $)=\left(U_{1}\right)^{(n-1)}$, the Cartan subgroup of the $S U_{n}$; (2) none of the non-perturbative $U_{1}$ 's mixes with any of the perturbative groups; (3) all twisted states are neutral under all the non-perturbative $U_{1}$ 's; and (4) all the non-perturbative $U_{1}$ 's have $\rho=0$. In addition, many $\mathbf{Z}_{4}$ and $\mathbf{Z}_{6}$ orbifolds have a locallyunbroken $E_{8}$ on the $\mathbf{Z}_{2}$ or $\mathbf{Z}_{3}$ fixed planes — and all such fixed planes carry invisible $U_{1}$ or $\left(U_{1}\right)^{2}$ gauge groups, exactly like similar fixed planes in the corresponding $\mathbf{Z}_{2}$ or $\mathbf{Z}_{3}$ models. For example, the $\mathbf{Z}_{6}$ orbifold of Section 4 has such an invisible $G_{7}=U_{1}$ on each of its $\mathbf{Z}_{2}$ fixed planes.

It is easy to see that any invisible $U_{1}$ gauge field must have $\rho=0$ for the sake of local anomaly cancellation. Indeed, an invisible seven-plane $U_{1}$ has no quantum anomalies since all the chiral fields are neutral — and no inflow anomalies — which involve only the ten-plane groups or the visible seven-plane groups that mix with them. Consequently, any invisible $U_{1}$ factor should also decouple from the intersection anomaly, which means it must have $\rho=0$. Unfortunately, this anomaly-counting argument does not tell us how the invisible seven-plane gauge groups differ from the visible seven-plane groups from the seven-dimensional point of view, so the M-theoretical origins of the $\rho_{\text {invisible }}=0$ remain obscure.

\subsection{Models with perturbative $U_{1}$ factors}

The invisible Abelian groups of the previous section are puzzling from the M-theoretical point of view, but as far as the local anomaly cancellation is concerned, the visible Abelian groups are much more troublesome. Generally, the combined quantum, inflow and intersection anomalies in such models cancel each other globally but not locally in $x^{11}$

\footnotetext{
15 There are ten distinct heterotic orbifolds of this type, including the standard $\mathbf{Z}_{2}$ orbifold, two different $\mathbf{Z}_{3}$ orbifolds, two $\mathbf{Z}_{4}$ orbifolds and five $\mathbf{Z}_{6}$ orbifolds.
} 


$$
\mathcal{A}(\mathrm{I} 6)+\mathcal{A}\left(\mathrm{I}^{\prime}\right)=0, \quad \text { but } \mathcal{A}(\mathrm{I} 6) \neq 0 \neq \mathcal{A}\left(\mathrm{I}^{\prime}\right) .
$$

In other words, the anomalies cancel locally in ten dimensions (which always works in any perturbative heterotic orbifold) but not the eleventh dimension.

As an example of such anomaly trouble, let us consider a $\mathbf{Z}_{4}$ orbifold summarized in Table 5.

Note that all data in Table 5 are completely determined by the perturbative heterotic string theory - except of course for the inherently non-perturbative $G_{7}$ and $Q_{7}$. However, the model must have $G_{7} \supset S U_{2} \times U(1)$ since the gauge couplings of the perturbative $S U_{2}$

Table 5

A $\mathbf{Z}_{4}$ orbifold with the $\left(S O_{10} \times S U_{4}\right) \times\left(E_{6} \times S U_{2} \times U_{1}\right)$ gauge group and anomaly troubles

Example 6: $\mathbf{Z}_{4}$ orbifold

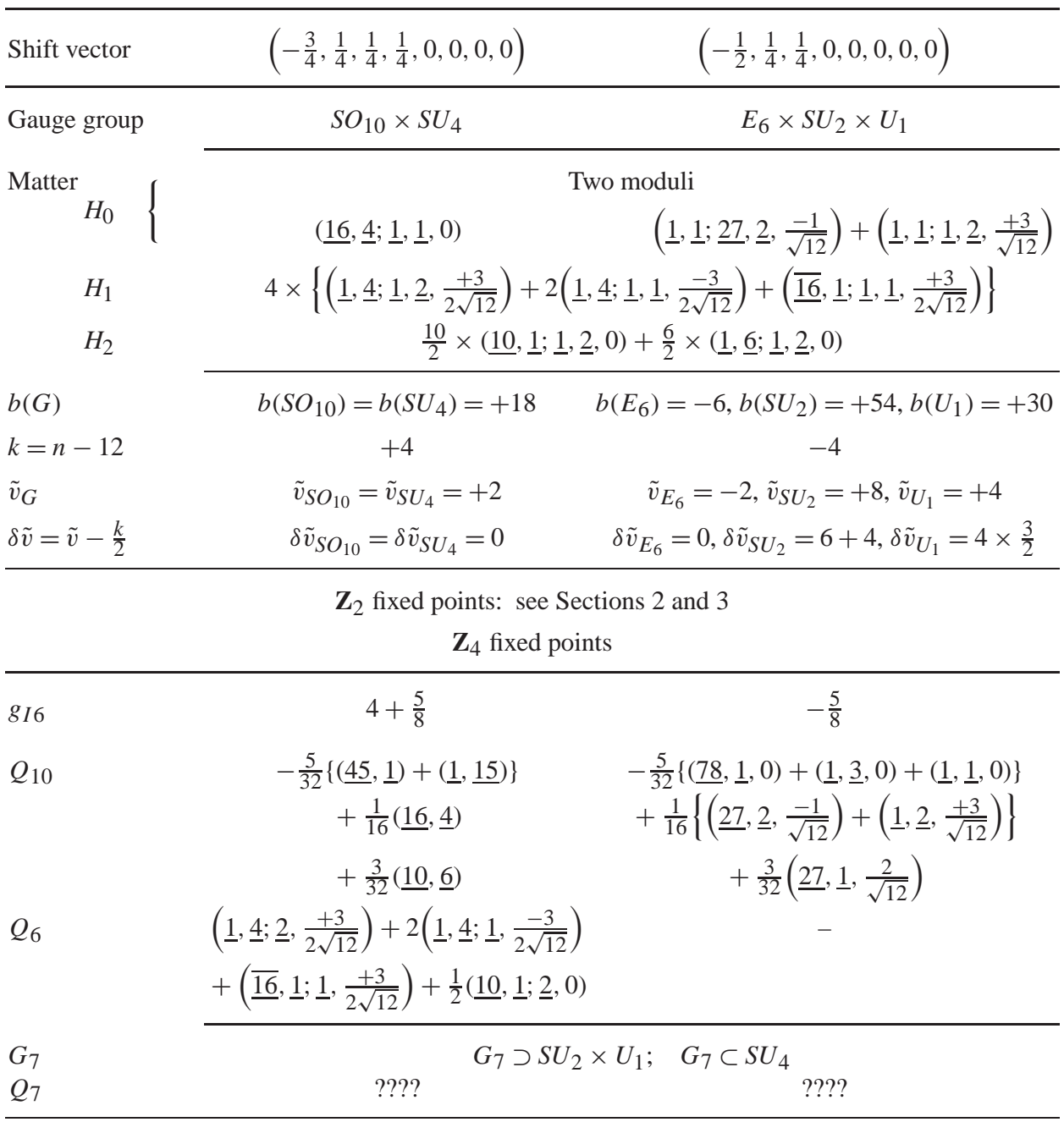


and $U_{1}$ group factors indicates their mixing with the non-perturbative gauge fields living on the $\mathbf{Z}_{4}$ fixed seven-planes. Actually, the $S U_{2}$ factor gets non-perturbative contributions from both $\mathbf{Z}_{2}$ and $\mathbf{Z}_{4}$ fixed planes, hence $\tilde{v}_{S U_{2}}-(k / 2)=6+4$. In the $U_{1}$ case however, only the $\mathbf{Z}_{4}$ fixed seven-planes are involved, but there appear to be a non-trivial mixing angle, thus $\tilde{v}_{U_{1}}=4 \times 3 / 2$ rather than simply 4 .

The question at this point is whether any consistent choice of $G_{7}$ and $Q_{7}^{(\prime)}$ would lead to a local cancellation of all the anomalies on the I6 and I6 $6^{\prime}$ planes. The answer turns out to be negative in an interesting way: the requirement of local anomaly cancellation does have a unique solution

$$
Q_{7}=-Q_{7}^{\prime}=-\frac{1}{2}(\underline{3}, 0)-\left(\underline{2}, \frac{3}{\sqrt{12}}\right) .
$$

In terms of the seven-plane fields and their boundary conditions, this means $G_{7}=S U_{3}$ $\times U_{1}^{\prime} \subset S U_{4}$ where $S U_{3} \supset S U_{2} \times U_{1}$ while the $U_{1}^{\prime}$ factor is invisible. In $S U_{3} \times U_{1}^{\prime}$ terms, $Q_{7}=\frac{1}{2}\left\{\underline{1}^{0}-\underline{8}^{0}\right\}$, which means free (Neumann) boundary conditions for all eight of $S U_{3}$ vector fields at I6 on the $S_{10} \times S U_{4}$ end of $x^{11}$. Consequently, the fields living on the I6 intersection plane should form complete multiplets of the $\left(\mathrm{SO}_{10} \times S U_{4}\right)^{\text {pert }} \times S U_{3}^{\text {n.p. }}$ gauge group visible at I6. Unfortunately, they do not - cf. the $Q_{6}$ row of the Table 5 which means the solution (5.2) to the local anomaly problems is inconsistent with group theory. Group-theoretically, the only possibilities consistent with all the quantum numbers are $G_{7}=S U_{2} \times U_{1}$ and $G_{7}=S U_{2} \times U_{1} \times U_{1}^{\prime}$. Both choices lead to non-zero net gauge, gravitational and mixed local anomalies on both the I6 and the I6' intersection planes.

This type of local anomaly miscancellation is common in orbifolds with perturbative abelian groups. Generally, the quantum numbers of the twisted states in such models require some fixed seven-planes to carry Abelian non-perturbative gauge fields that lock onto the perturbative $U_{1}$ fields on one side of the eleventh dimension, say at I6, but have free boundary conditions on the other side at I6'. Locally on the I6 $6^{\prime}$ plane, there are no $F_{U_{1}}^{4}$ inflow or intersection anomalies (since the $U_{1}$ does not lock onto the ten-plane gauge fields on that side) but there is a non-zero quantum $F_{U_{1}}^{4}$ anomaly because there are $U_{1}$ charged hyper-multiplets (twisted states) but no charged vector-multiplets (if there were charged vector fields, the group would not be Abelian). Altogether, the Abelian gauge anomaly $F_{U_{1}}^{4}$ does not cancel locally on the I6' plane.

'Experimentally', in all the models we have studied, a miscancelled Abelian gauge anomaly is accompanied by miscancelled anomalies involving other non-perturbative gauge fields living on the same plane as well as gravity. On the other hand, all anomalies involving the un-mixed purely perturbative gauge fields do cancel out. Algebraically,

$$
\mathcal{A}(\mathrm{I} 6)=-\mathcal{A}\left(\mathrm{I}^{\prime}\right)=P\left(F_{7}, R\right)
$$

for some quartic polynomial $P$ in seven-dimensional gauge and gravitational curvature two-forms $F_{7}$ and $R$.

The algebraic structure (5.3) of the net local anomaly suggests that it may ultimately cancel against a Chern-Simons action term in the effective theory on the fixed seven-plane. Indeed, consider 


$$
\text { Action } \supset \int_{7 \mathrm{P}} I_{7},
$$

where $I_{7}$ is a Chern-Simons 7-form which varies under gauge transformations by a total derivative, $\delta I_{7}=d I_{6}^{1}$. Consequently,

$$
\delta \text { Action }=\int_{\partial(7 \mathrm{P})} I_{6}^{1}=\int_{\mathrm{I}^{\prime}} I_{6}^{1}-\int_{\mathrm{I} 6} I_{6}^{1},
$$

which looks exactly like local anomalies on the I6 and I6' boundary six-planes with exactly opposite anomaly polynomials

$$
I_{8}\left(\mathrm{I}^{\prime}\right)=-I_{8}(\mathrm{I} 6)=d I_{7}
$$

Therefore, if $d I_{7}=P\left(F_{7}, R\right)$ (cf. Eq. (5.3)) or rather $d I_{7}=P /\left(192 \pi^{3} i\right)$, then all anomalies would cancel locally.

We are currently investigating whether such Chern-Simons terms do actually cancel the residual local anomalies of orbifold models with Abelian gauge factors. There are (at least) two issues that must be addressed. First, there is a question of normalization: gauge, gravitational and mixed Chern-Simons terms all have quantized coefficients, which may turn out to be consistent or inconsistent with the local anomaly cancellation in various models. The second open problem is the physical origin of the $7 \mathrm{~d}$ Chern-Simons terms. All anomalies cancel locally in models considered in Sections 2-4 without any help from the CS terms, so why should the CS terms appear in other models? At the moment the CS terms still seem to pose as many new questions as they are able to answer. We hope to be able to report some progress on this issue in the near future.

\section{Summary and discussion}

In this paper we have investigated the correspondence between perturbative heterotic $E_{8} \times E_{8}$ orbifolds on $T^{4} / \mathbf{Z}_{N}(N=2,3,4,6)$ and their M-theory duals. Our aim was to obtain information about the structure of M-theory orbifolds, in particular the spatial locations of all the charged fields and the gauge groups that act upon them. Our main tools were constraints arising from local consistency of quantum numbers, from the gauge coupling considerations and from the requirements of local anomaly cancellation. We have put forward a scenario which allowed us to resolve an apparent paradox associated with the existence of twisted massless states simultaneously charged under gauge groups which appear to live on two different boundary ten-planes. The resolution relies on the mixing of a factor (or factors) of the gauge group $G$ living on one boundary ten-plane with the $G_{7}$ gauge groups living on the fixed seven-planes $R^{5,1} \otimes\{$ f.p. $\} \otimes S^{1} / \mathbf{Z}_{2}$ of the orbifold. The twisted states which live on the I6 $6^{\prime}$ planes where the fixed seven-planes reach the other ten-plane boundary thus have perfectly local $G_{7} \times G^{\prime}$ charges. Thanks to mixing, however, the $G_{7}$ charges of those states masquerade as the perturbative $G$ charges from the sixdimensional point of view, hence the appearance of the simultaneous $G$ and $G^{\prime}$ charges in the heterotic perturbation theory. 
Considerations of the gauge couplings confirm that there is a mixing between the nonperturbative gauge groups on the seven-planes and the perturbative gauge group on the boundary planes. Another confirmation comes from the overall $6 \mathrm{~d}$ anomaly polynomial. The most powerful test however is the local anomaly cancellation. Here the correct allocation of all states - in particular the hyper and vector component of the seven-plane gauge multiplets - to the six-planes where the anomaly is required to cancel is absolutely crucial. A consistent assignment of states was only possible after a judicious choice of boundary conditions on the seven-plane fields which projects out the vector components on one side and the hyper-component on the other. This also guaranteed that there are no additional massless states in the $R_{11} \rightarrow 0$ heterotic limit.

There are three contributions to the net local anomaly: quantum anomalies, inflow anomalies and intersection anomalies. Given a correct identification of all the locally relevant fields, the quantum one-loop anomalies are fairly straightforward, but the inflow and intersection anomalies involve un-settled issues of the overall normalization. In principle, the overall normalization of the inflow anomalies follows from that of the ChernSimons terms in eleven-dimensional SUGRA, but the derivation is rather subtle and the result is controversial. Instead, we simply calibrated the normalization by requiring local anomaly cancellation in the $\mathbf{Z}_{2}$ model (cf. Section 3), then used the same normalization in all the other models of Section 4; eventually, this normalization ought to be confirmed by a direct derivation.

Likewise, the $\eta$ and $\rho$ parameters of the intersection anomaly (cf. Eqs. (3.11) and (3.12)) should follow from the seven-dimensional Chern-Simons terms (which in turn follow from the M-theory) and indeed the analysis of Section 4.4 gives specific values $\eta=T(1)$ and $\rho=1$. Alternatively, we can treat them as free parameters, fixed by the anomaly cancellation requirements of each model. According to Eqs. (4.17), $\eta=T$ (1) is indeed universally valid, but the $\rho$ parameter is more tricky: $\rho=1$ works for model with $G_{7}=S U_{n}$ (cf. Sections 3 and 4) but models with the invisible Abelian $G_{7}=U_{1}^{(n-1)}$ (cf. Section 5.1) require $\rho=0$ instead. Unfortunately, we do not know yet the M-theoretic origin of the seven-plane $S U_{n}$ breakdown to its Cartan subgroup in these models, and we do not know how this Cartan subgroups ends up with $\rho=0$ either.

A worse anomaly trouble plagues models with Abelian factors in their perturbative gauge groups, cf. Section 5.2. In those models, the local anomalies simply do not cancel for any consistent choices of the seven-plane gauge fields and their boundary conditions at the two ends of the $x^{11}$. This presumably means one of the two things: either we do not know how to correctly interpret such models in M-theory terms or else there must be additional sources of local anomalies. For example, a Chern-Simons term in the seven-plane effective action has an effect of transferring local $6 \mathrm{~d}$ anomaly from one boundary of the seven plane to the other boundary, thus possibly helping to cancel the local anomalies on both boundaries. Much work is needed however before we know whether this mechanism really works.

Our main conclusion is that the heterotic orbifolds do have consistent M-theory duals with identical massless spectra and gauge couplings and locally cancelled anomalies. Unfortunately, while the duality and the local anomalies tell us what should happen on 
every six-, seven- or ten-plane of the dual model, we do not understand how it happens in M-theory. The big mystery is the dynamic origin of the boundary conditions for the seven-plane fields. In particular, we know that each seven-plane field always has exactly opposite boundary conditions on the two boundaries of the 7P, but the M-theoretic reasons for this twist remain completely obscure. Another mystery why the $\mathbf{Z}_{n}$-fixed seven-planes carry full $S U_{n}$ gauge groups in some models while in other models the $S U_{n}$ is broken to a subgroup; in fact, we are not even sure of the mechanism of such seven-plane symmetry breaking. We are however quite certain that the eventual resolution of these issues will shed much new light on the basic structure of the M-theory.

We conclude this article with a few comments on M-theory duals of the four-dimensional heterotic orbifolds. Generally, such models live on $\mathbb{R}^{3,1} \otimes\left(T^{6} / \Gamma\right) \otimes\left(S^{1} / \mathbf{Z}_{2}\right)$ comprising the $11 \mathrm{~d}$ bulk, fixed seven-planes, fixed five-planes as well as their respective ten-plane, sixplane and four-plane boundaries. The bulk, the seven-planes and their boundaries should behave just as they do in the $6 \mathrm{~d}$ orbifolds (modulo orbifolding of their extra compact coordinates), it's the five-planes that radically complicate the physics. The problem with the fixed five-planes is that they are poorly understood from the M-theory point of view. We know that the singular five-planes in M-theory carry superconformal theories whose excitations include both massless particles and tensionless strings. Generally, the superconformal theories in five dimensions are associated with infinite-coupling gauge theories, but the M-theory tells us nothing about the gauge group, only the global symmetries (if any) and some gauge-invariant operators - and these data are quite insufficient for the model building purposes. Also, the constraints of local anomaly cancellation on the four-plane boundaries of the five-planes are rather weak, simply because the anomalies in four dimensions are much simpler than in six. Consequently, although we have tentatively identified the M-theory duals of a few $4 \mathrm{~d}$ heterotic orbifolds, we do not have enough constraints to be confident in our identification at the time of this writing. This work is in progress and we hope to present some interesting results in the near future.

\section{Acknowledgements}

An important part of the project was accomplished while V.K., J.S. and S.Y. visited Universität München. They would like to thank the Sektion Physik for its hospitality. S.Y. would also like to thank the theory group of CERN. S.T. would like to thank R. Minasian and C. Scrucca for helpful discussions.

\section{Appendix A. Anomaly polynomials for $\mathcal{N}=1$ multiplets in $d=6$}

This appendix summarizes the six-dimensional gravitational, gauge and mixed anomaly contributions of all relevant six-dimensional $\mathcal{N}=1$ multiplets, see, e.g., [15]. The representation content of each such multiplet is spelled out in terms of the little group 
$S O_{4} \simeq S U_{2} \times S U_{2}$ for massless particles in $6 \mathrm{~d}$. The correctly normalized anomaly polynomials are

$$
I_{8}=\frac{-i}{4 !(2 \pi)^{3}} \mathcal{A},
$$

but since we use $\mathcal{A}$ instead of $I_{8}$ throughout this paper, the anomalies below are written in terms of $\mathcal{A}$ as well.

- Gravity multiplet $[(3,3)+2(2,3)+(1,3)]$ :

$$
\mathcal{A}_{\text {grav }}=-\frac{273}{240} \operatorname{tr} R^{4}+\frac{51}{192}\left(\operatorname{tr} R^{2}\right)^{2} .
$$

tr is the trace in the vector representation of $S O(6)$.

- Vector-multiplets $n_{V}[(2,2)+2(1,2)]$ :

$$
\mathcal{A}_{\mathrm{V}}=-\frac{n_{\mathrm{V}}}{240} \operatorname{tr} R^{4}-\frac{n_{\mathrm{V}}}{192}\left(\operatorname{tr} R^{2}\right)^{2}+\frac{1}{4} \operatorname{tr} R^{2} \operatorname{Tr}_{\mathrm{V}} F^{2}-\operatorname{Tr}_{\mathrm{V}} F^{4}
$$

since the vector multiplets comprise the adjoint representation of the gauge group $G, \operatorname{Tr}_{V}$ is the trace in the adjoint representation and $n_{V}=\operatorname{dim}(G)$.

- Hyper-multiplets $n_{H}[2(2,1)+4(1,1)]$ :

$$
\mathcal{A}_{\mathrm{H}}=\frac{n_{\mathrm{H}}}{240} \operatorname{tr} R^{4}+\frac{n_{\mathrm{H}}}{192}\left(\operatorname{tr} R^{2}\right)^{2}-\frac{1}{4} \operatorname{tr} R^{2} \operatorname{Tr}_{\mathrm{H}} F^{2}+\operatorname{Tr}_{\mathrm{H}} F^{4},
$$

where $\operatorname{Tr}_{H}$ is the trace in the representation of $G$ comprised of all the hypermultiplets (whatever such representation might be in any particular model) and $n_{H}$ is the net number of hypermultiplets in the model.

- Selfdual-tensor multiplet $[(3,1)+2(2,1)+(1,1)]$ :

$$
\mathcal{A}_{\text {tensor }}=\frac{29}{240} \operatorname{tr} R^{4}-\frac{7}{192}\left(\operatorname{tr} R^{2}\right)^{2} \text {. }
$$

\section{Appendix B. Further anomaly considerations}

In Section 2.2, we mentioned that in perturbative $\mathrm{K} 3$ compactifications of the heterotic string, the gauge couplings are related to the $6 \mathrm{~d}$ anomaly polynomial. In this appendix, we explain this relation.

The anomaly we are concerned with here is the overall anomaly of the $6 \mathrm{~d}$ effective theory rather than local anomalies on some singular six-planes. In perturbative K3 compactifications, there is just one tensor field $B_{\mu \nu}$, hence the Green-Schwarz mechanism of anomaly cancellation works only if the net anomaly happens to factorize in the form [8]:

$$
\frac{2}{3} \mathcal{A}=\left(\operatorname{tr} R^{2}-\sum_{\alpha} v_{\alpha} \operatorname{tr} F_{\alpha}^{2}\right) \wedge\left(\operatorname{tr} R^{2}-\sum_{\alpha} \tilde{v}_{\alpha} \operatorname{tr} F_{\alpha}^{2}\right) \equiv I_{4} \wedge \tilde{I}_{4} .
$$

Furthermore, the $\mathcal{N}=1, d=6$ supersymmetry relates the couplings of the $B_{\mu v}$ tensor fields to those of the heterotic dilaton $\phi$, hence the factorization coefficient $v_{\alpha}$ and $\tilde{v}_{\alpha}$ in this formula also show up in the exact Eqs. (2.2) for the gauge couplings. 
The Green-Schwarz mechanism does not cancel the irreducible $\operatorname{tr} R^{4}$ term in the anomaly polynomial, hence anomaly-free theories with just one selfdual-tensor multiplet must have $n_{H}-n_{V}=244$, cf. individual multiplets' anomalies listed in Appendix A. Consequently, the net anomaly can be summarized as

$$
\frac{2}{3} \mathcal{A}=\left(\operatorname{tr} R^{2}\right)^{2}-\frac{1}{6} \operatorname{tr} R^{2} \operatorname{Tr}_{H-V} F^{2}+\frac{2}{3} \operatorname{Tr}_{H-V} F^{4},
$$

were the notation $\operatorname{Tr}_{H-V}$ denotes the trace taken over all hyper-multiplets minus the trace over all vector-multiplets. The relative minus-sign follows from opposite chiralities of fermions in $\mathcal{N}=1, d=6$ hyper- and vector-multiplets. Comparing the mixed gauge/gravitational anomaly terms in Eqs. (B.2) and (B.1), we see that Green-Schwarz anomaly cancellation requires

$$
\operatorname{Tr}_{H-V} F^{2}=\sum_{\alpha} 6\left(v_{\alpha}+\tilde{v}_{\alpha}\right) \operatorname{tr} F_{\alpha}^{2} .
$$

(See Appendix C for exact normalizations of various traces.) Curiously, $\operatorname{Tr}_{H-V} F^{2}$ also appears in beta-function coefficients of $\mathcal{N}=2, d=4$ gauge theories (including but not limited to toroidal compactifications of the $\mathcal{N}=1, d=6$ theories); specifically, $\operatorname{Tr}_{H-V} F^{2}=\sum_{\alpha} b_{\alpha} \operatorname{tr} F_{\alpha}^{2}$. Comparing this formula to Eq. (B.3), we immediately arrive at Eq. (2.3).

From the M-theory point of view, there is another interesting way to write the anomaly polynomial as a sum of two factorized terms, each associated with the boundary at the corresponding end of $x^{11}$. For smooth K3 compactifications (perturbative or otherwise) with instanton numbers $n_{1}+n_{2}=24$ (hence exactly one selfdual-tensor multiplet), we have [16]

$$
\begin{aligned}
\frac{2}{3} \mathcal{A}= & \left(\frac{1}{2} \operatorname{tr} R^{2}-\sum_{\alpha} v_{\alpha 1} \operatorname{tr} F_{\alpha 1}^{2}\right) \wedge\left(\frac{1}{4}\left(n_{1}-8\right) \operatorname{tr} R^{2}-\sum_{\alpha} \tilde{v}_{\alpha 1} \operatorname{tr} F_{\alpha 1}^{2}\right) \\
& +\left(\frac{1}{2} \operatorname{tr} R^{2}-\sum_{\alpha} v_{\alpha 2} \operatorname{tr} F_{\alpha 2}^{2}\right) \wedge\left(\frac{1}{4}\left(n_{2}-8\right) \operatorname{tr} R^{2}-\sum_{\alpha} \tilde{v}_{\alpha 2} \operatorname{tr} F_{\alpha 2}^{2}\right) .
\end{aligned}
$$

There are similar expressions for singular K3's such as orbifolds. For example, the $\mathbf{Z}_{2}$ model of Section 2 has

$$
\begin{aligned}
\frac{2}{3} \mathcal{A}= & \left(\frac{1}{2} \operatorname{tr} R^{2}-\operatorname{tr} F_{E_{7}}^{2}-\operatorname{tr} F_{S U_{2}}^{2}\right) \wedge\left(2 \operatorname{tr} F_{E_{7}}^{2}-14 \operatorname{tr} F_{S U_{2}}^{2}\right) \\
& +\left(\frac{1}{2} \operatorname{tr} R^{2}-\operatorname{tr} F_{S O_{16}}^{2}\right) \wedge\left(2 \operatorname{tr} R^{2}-2 \operatorname{tr} F_{S O_{16}}^{2}-16 \operatorname{tr} F_{S U_{2}}^{2}\right) .
\end{aligned}
$$

We observe that one of the gauge factors, namely $S U_{2}$, now appears on both sides but only non-perturbatively $(v=0, \tilde{v}=16)$ on the $S O_{16}$ side. This lends support to the M-theory description of this model that we have put forward in Section 2. We have a nonperturbatively generated gauge group $\left(S U_{2}\right)^{16}$. Each of these $S U_{2}$ 's has $\tilde{v}=1$ and they mix with the perturbative $S U_{2}$ such that only the diagonal $S U_{2}$ contributes in the heterotic description of the model, i.e., only one $S U_{2}$ is visible. This $S U_{2}$ is however visible on both sides of the $x^{11}$ interval. 


\section{Appendix C. Some results from group theory}

We collect some group theoretical results which are needed to verify anomaly cancellation. The notation is such that $\operatorname{Tr}_{R}$ is always the trace in the representation $R$ whereas $\operatorname{tr} F^{2}$ means $\sum_{a} F^{a} F^{a}$. The normalizations are such that the long roots are normalized to length 1 .

$\mathrm{SU}_{2}$

$$
\begin{array}{ll}
\operatorname{Tr}_{\underline{2}} F^{2} \equiv \frac{1}{2} \operatorname{tr} F^{2}, & \operatorname{Tr}_{\underline{2}} F^{4}=\frac{1}{8}\left(\operatorname{tr} F^{2}\right)^{2}, \\
\operatorname{Tr}_{\underline{3}} F^{2}=2 \operatorname{tr} F^{2}, & \operatorname{Tr}_{\underline{3}} F^{4}=2\left(\operatorname{tr} F^{2}\right)^{2},
\end{array}
$$

$\mathrm{SU}_{3}$

$$
\begin{array}{ll}
\operatorname{Tr}_{\underline{3}} F^{2}=\frac{1}{2} \operatorname{tr} F^{2}, & \operatorname{Tr}_{\underline{3}} F^{4}=\frac{1}{8}\left(\operatorname{tr} F^{2}\right)^{2}, \\
\operatorname{Tr}_{\underline{8}} F^{2}=3 \operatorname{tr} F^{2}, & \operatorname{Tr}_{\underline{8}} F^{4}=\frac{9}{4}\left(\operatorname{tr} F^{2}\right)^{2},
\end{array}
$$

$S U_{N}, N \geqslant 4$

$$
\begin{aligned}
& \operatorname{Tr}_{\underline{N}} F^{2}=\frac{1}{2} \operatorname{tr} F^{2}, \quad \operatorname{Tr}_{\underline{N}} F^{4}=\frac{3}{16}\left(\operatorname{tr} F^{2}\right)^{2}-\frac{1}{4} \operatorname{tr} F^{4}, \\
& \operatorname{Tr}_{\mathrm{ad}} F^{2}=N \operatorname{tr} F^{2}, \quad \operatorname{Tr}_{\mathrm{ad}} F^{4}=\frac{3}{8}(N+4)\left(\operatorname{tr} F^{2}\right)^{2}-\frac{N}{2} \operatorname{tr} F^{4}, \\
& \operatorname{Tr}_{\mathrm{B}} F^{2}=\frac{N-2}{2} \operatorname{tr} F^{2}, \\
& \operatorname{Tr}_{\mathrm{B}} F^{4}=\frac{3}{16}(N-4)\left(\operatorname{tr} F^{2}\right)^{2}+\left(2-\frac{N}{4}\right) \operatorname{tr} F^{4}, \\
& \operatorname{Tr}_{\mathrm{B}} F^{2}=\frac{1}{4}(N-2)(N-3) \operatorname{tr} F^{2}, \\
& \operatorname{Tr}_{\mathrm{O}} F^{4}=\frac{3}{32}\left(N^{2}-9 N+22\right)\left(\operatorname{tr} F^{2}\right)^{2}-\frac{1}{8}\left(N^{2}-17 N+54\right) \operatorname{tr} F^{4},
\end{aligned}
$$

$S O_{N}, N \geqslant 5$

$$
\begin{array}{ll}
\operatorname{Tr}_{\underline{N}} F^{2} \equiv \operatorname{tr} F^{2}, & \operatorname{Tr}_{\underline{N}} F^{4} \equiv \operatorname{tr} F^{4}, \\
\operatorname{Tr}_{\text {ad }} F^{2}=(N-2) \operatorname{tr} F^{2}, & \operatorname{Tr}_{\text {ad }} F^{4}=3\left(\operatorname{tr} F^{2}\right)^{2}+(N-8) \operatorname{tr} F^{4}, \\
\operatorname{Tr}_{\text {spinor }} F^{2}=\frac{\mathrm{d}}{8} \operatorname{tr} F^{2}, & \operatorname{Tr}_{\text {spinor }} F^{4}=\frac{\mathrm{d}}{4}\left[\frac{3}{16}\left(\operatorname{tr} F^{2}\right)^{2}-\frac{1}{4} \operatorname{tr} F^{4}\right],
\end{array}
$$

where $\mathrm{d}=2^{\frac{N}{2}-1}$ ( $N$ even) and $\mathrm{d}=2^{\frac{N-1}{2}}(N$ odd $)$ is the dimension of the spinor representation.

$E_{6}$

$$
\begin{array}{ll}
\operatorname{Tr}_{\underline{27}} F^{2} \equiv 3 \operatorname{tr} F^{2}, & \operatorname{Tr}_{\underline{27}} F^{4}=\frac{3}{4}\left(\operatorname{tr} F^{2}\right)^{2}, \\
\operatorname{Tr}_{\underline{78}} F^{2}=12 \operatorname{tr} F^{2}, & \operatorname{Tr}_{\underline{78}} F^{4}=\frac{9}{2}\left(\operatorname{tr} F^{2}\right)^{2},
\end{array}
$$


$E_{7}$

$$
\begin{array}{ll}
\operatorname{Tr}_{\underline{56}} F^{2} \equiv 6 \operatorname{tr} F^{2}, & \operatorname{Tr}_{\underline{56}} F^{4}=\frac{3}{2}\left(\operatorname{tr} F^{2}\right)^{2}, \\
\operatorname{Tr}_{\underline{133}} F^{2}=18 \operatorname{tr} F^{2}, & \operatorname{Tr}_{\underline{133}} F^{4}=6\left(\operatorname{tr} F^{2}\right)^{2},
\end{array}
$$

$E_{8}$

$$
\operatorname{Tr}_{\mathrm{ad}} F^{2}=30 \operatorname{tr} F^{2}, \quad \operatorname{Tr}_{\mathrm{ad}} F^{4}=9\left(\operatorname{tr} F^{2}\right)^{2} .
$$

\section{References}

[1] E. Witten, String dynamics in various dimensions, Nucl. Phys. B 443 (1995) 85, hep-th/ 9503124.

[2] A. Sen, An introduction to non-perturbative string theory, hep-th/9802051.

[3] P.K. Townsend, Four lectures on M-theory, hep-th/9612121.

[4] P. Hořava, E. Witten, Eleven-dimensional supergravity on a manifold with boundary, Nucl. Phys. B 475 (1996) 94, hep-th/9603142.

[5] P. Hořava, E. Witten, Heterotic and type I string dynamics from eleven dimensions, Nucl. Phys. B 460 (1996) 506, hep-th/9510209.

[6] G. Aldazabal, A. Font, L. Ibanez, F. Quevedo, Chains of $N=2, D=4$ heterotic/type II duals, Nucl. Phys. B 461 (1996) 85, hep-th/9510093.

[7] J. Erler, Anomaly cancellation in six dimensions, J. Math. Phys. 35 (1994) 1819, hep-th/ 9304104.

[8] M. Duff, R. Minasian, E. Witten, Evidence for heterotic/heterotic duality, Nucl. Phys. B 465 (1996) 413, hep-th/9601036.

[9] M. Berkooz, R.G. Leigh, J. Polchinski, J.H. Schwarz, N. Seiberg, E. Witten, Anomalies, dualities, and topology of $D=6 N=1$ superstring vacua, Nucl. Phys. B 475 (1996) 115, hep-th/9605184.

[10] L. Alvarez-Gaume, E. Witten, Gravitational anomalies, Nucl. Phys. B 234 (1984) 269.

[11] M. Faux, D. Lüst, B. Ovrut, Intersecting orbifold planes and local anomaly cancellation in M-theory, Nucl. Phys. B 554 (1999) 437, hep-th/9903028.

[12] G. Aldazabar, A. Font, L. Ibanez, A. Uranga, G. Violero, Non-perturbative heterotic $D=6$, $N=1$ orbifold vacua, Nucl. Phys. B 519 (1998) 239, hep-th/9706158.

[13] S. Stieberger, $(0,2)$ heterotic gauge couplings and their M-theory origin, Nucl. Phys. B 541 (1999) 109, hep-th/9807124.

[14] A. Sen, Orbifolds of M-theory and string theory, Mod. Phys. Lett. A 11 (1996) 1339, hep-th/ 9603113.

[15] M. Green, J. Schwarz, E. Witten, Superstring Theory, Vol. II, CUP, 1987.

[16] N. Seiberg, E. Witten, Comments on string dynamics in six dimensions, Nucl. Phys. B 471 (1996) 121, hep-th/9603003. 